\title{
Modeling close encounters with massive asteroids: a Markovian approach ${ }^{\star}$ An application to the Vesta family
}

\author{
V. Carruba ${ }^{1,4}$, F. Roig ${ }^{2}$, T. A. Michtchenko ${ }^{1}$, S. Ferraz-Mello ${ }^{1}$, and D. Nesvorný ${ }^{3}$ \\ 1 IAG, Universidade de São Paulo, São Paulo, SP 05508-900, Brazil \\ e-mail: [valerio; tatiana;sylvio]@astro.iag.usp.br \\ 2 Observatório Nacional, Rio de Janeiro, RJ 20921-400, Brazil \\ e-mail: froig@on.br \\ 3 Southwest Research Institute, Department of Space Studies, Boulder, Colorado 80302 \\ e-mail: davidn@boulder.swri.edu \\ 4 Now at Departamento de Física e Astronomia, UNIVAP, Sâo José dos Campos, SP 12244-000, Brazil \\ e-mail: valerio@univap.br \\ Received 17 July 2006 / Accepted 6 December 2006
}

ABSTRACT

\begin{abstract}
Context. Nearly all members of the Vesta family cross the orbits of (4) Vesta, one of the most massive asteroids in the main belt, and some of them approach it closely. When mutual velocities during such close encounters are low, the trajectory of the small body can be gravitationally deflected, consequently changing its heliocentric orbital elements. While the effect of a single close encounter may be small, repeated close encounters may significantly change the proper element distribution of members of asteroid families.

Aims. We develop a model of the long-term effect of close encounters with massive asteroids, so as to be able to predict how far former members of the Vesta family could have drifted away from the family.

Methods. We first developed a new symplectic integrator that simulates both the effects of close encounters and the Yarkovsky effect. We analyzed the results of a simulation involving a fictitious Vesta family, and propagated the asteroid proper element distribution using the probability density function ( $p d f$ hereafter), i.e. the function that describes the probability of having an encounter that modifies a proper element $x$ by $\Delta x$, for all the possible values of $\Delta x$. Given any asteroids' proper element distribution at time $t$, the distribution at time $t+T$ may be predicted if the $p d f$ is known (Bachelier 1900, Théorie de la spéculation; Hughes 1995, Random Walks and Random Environments, Vol. I).

Results. We applied our new method to the problem of V-type asteroids outside the Vesta family (i.e., the 31 currently known asteroids in the inner asteroid belt that have the same spectral type of members as the Vesta family, but that are outside the limits of the dynamical family) and determined that at least ten objects have a significant diffusion probability over the minimum estimated age of the Vesta family of 1.2 Gyr (Carruba et al. 2005, A\&A, 441, 819). These objects can therefore be explained in the framework of diffusion via repeated close encounters with (4) Vesta of asteroids originally closer to the parent body.

Conclusions. We computed diffusion probabilities at the location of four of these asteroids for various initial conditions, parametrized by values of initial ejection velocity $V_{\mathrm{ej}}$. Based on our results, we believe the Vesta family age is (1200 \pm 700$)$ Myr old, with an initial ejection velocity of $(240 \pm 60) \mathrm{m} / \mathrm{s}$.
\end{abstract}

Key words. minor planets, asteroids - celestial mechanics

\section{Introduction}

Nearly all bodies in the asteroid belt cross the orbits of (1) $\mathrm{Ceres}^{1}$, (2) Pallas, (4) Vesta, or (10) Hygiea, and some approach them closely. When mutual encounter velocities are low, the trajectory of a small body making a close fly-by of these much larger asteroids can be gravitationally deflected, with a consequent change in its heliocentric orbital elements. While the probabilities and typical mutual velocities of such encounters are well understood (Farinella \& Davis 1992; Bottke \& Greenberg 1993), only a few recent studies have been carried out on their long-term effects (Nesvorný et al. 2002; Carruba et al. 2003).

\footnotetext{
* Appendices are only available in electronic form at http://www. aanda.org

1 By the time this article was accepted for publication the state of (1) Ceres had changed, so this body is now considered a dwarf planet by the International Astronomical Union (IAU). For the sake of simplicity we will continue to refer to Ceres as a massive asteroid in this work.
}

While the effect of a single encounter might be small, repeated close encounters with large asteroids can produce significant changes in asteroids' proper elements over long time scales $(\simeq 1 \mathrm{Gyr})$. Also, in contrast to the Yarkovsky effect that mostly modifies the asteroid's semimajor axis and is inversely proportional to the asteroid size, close encounters with massive asteroids are only weakly size-dependent and are also able to significantly modify the proper eccentricity $e$ and inclination $i$ of bodies.

We investigated the role that close encounters may have played in changing the proper elements of members of asteroid families, and, in particular, we concentrate our attention on the Vesta family. For this task, we wrote a new code that simulates both the effect of close encounters of massless particles with massive asteroids and the diurnal and seasonal versions of the Yarkovsky effect. To extrapolate the results of our numerical simulations, we developed a new method based on computing the probability distribution function for the changes in 


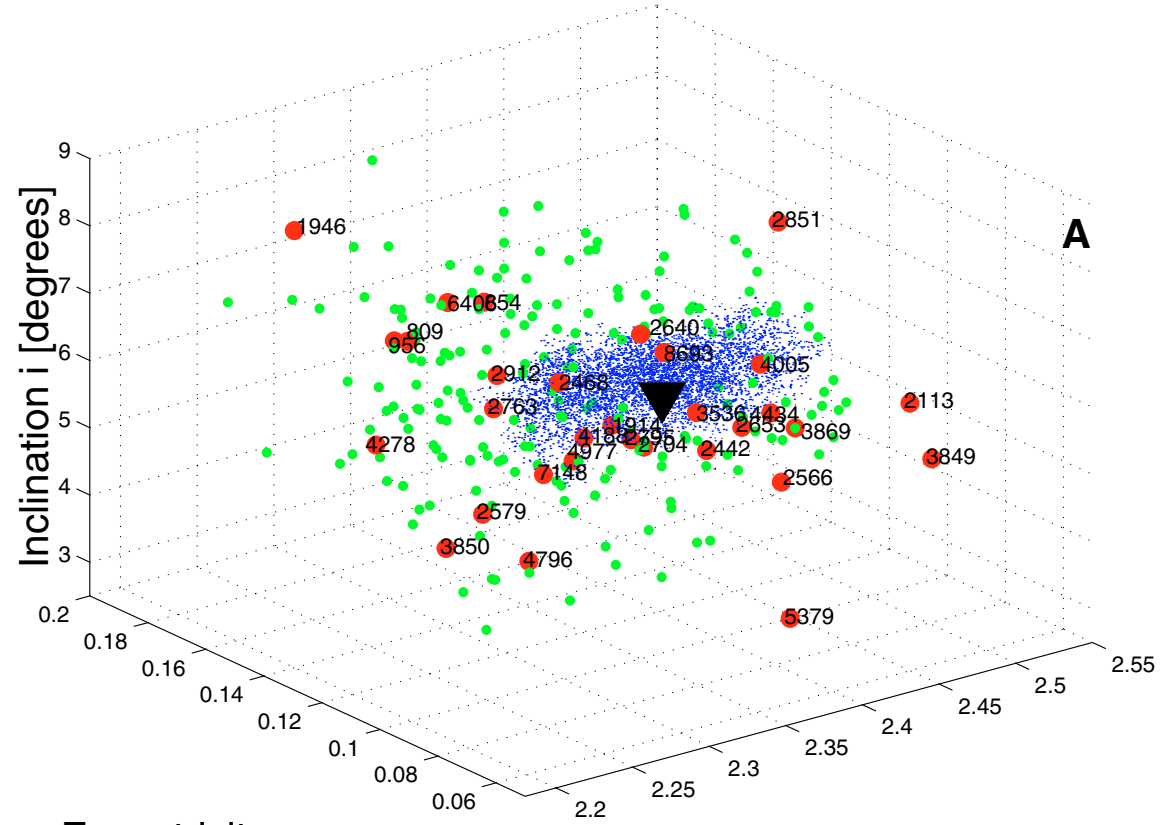

Eccentricity e
Semimajor axis a $[\mathrm{AU}]$
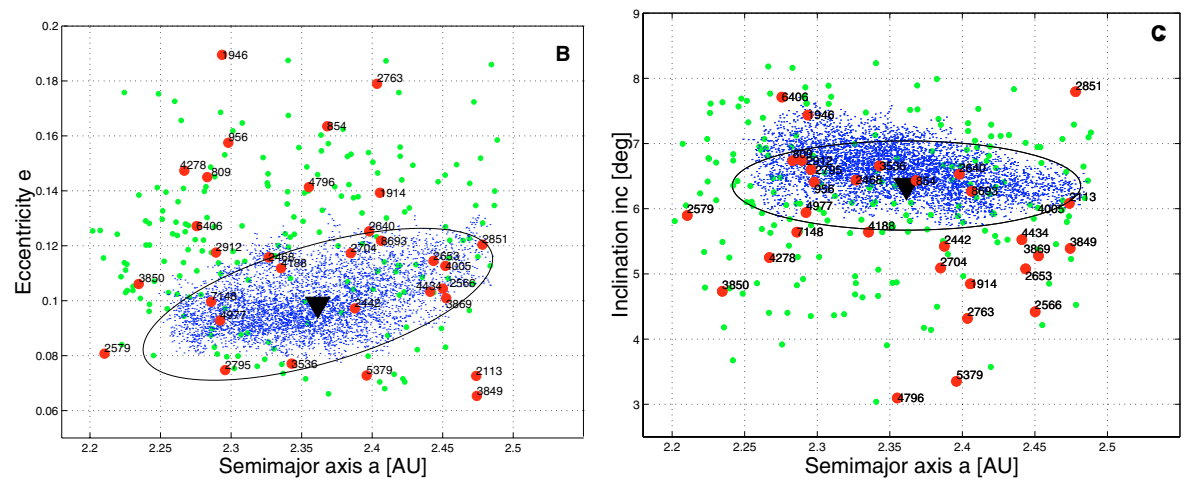

Fig. 1. Location in proper element space (a-e-i projection, A), a-e projection, B), and $a-i$ projection, C)) of the 5112 asteroids (small dots) members of the Vesta family (Mothè-Diniz et al. 2005). The triangle shows the location of (4) Vesta itself, while the ellipse in Figs. 1b,c displays the $450 \mathrm{~m} / \mathrm{s}$ level of maximum velocity at infinity (computed with Eqs. (5)-(7) in Carruba et al. (2003), see also footnote 1). The large numbered dots show the locations of the $31 \mathrm{~V}$-type asteroids that are not members of the family (see Table 4 for the asteroid identification). The unnumbered dots show the locations of the SLOAN candidates (Roig \& Gil-Hutton 2006). proper elements due to encounters with (4) Vesta (Bachelier 1900; Hughes 1995).

In our previous article (Carruba et al. 2005), we discussed the problem of V-type asteroids outside the Vesta family ${ }^{2}$, which are usually associated with a basaltic surface composition (McCord 1970; Bus 2002; Duffard et al. 2004). Until the work of Xu et al. (1995), the discovery of 1459 Magnya (Lazzaro 2000) and of several V-type NEAs (Bus \& Binzel 2002, and references therein), the only known V-type asteroids were members of the Vesta family. Xu et al. (1995) identified the first V-type asteroid (2579 Spartacus) in the inner belt that is not a member of the Vesta family, and, more recently, Florczak et al. (2002) identified two other inner belt relatively large asteroids located well outside the edges of the family (809 Lundia and 956 Elisa) that

2 The Vesta family is identified using the hierarchical clustering method (HCM, see Bendjoya \& Zappalá 2002, and references therein) to the proper element database of numbered and multiopposition objects avalaible through the AstDys website (http://hamilton.dm. unipi.it/astdys, see Carruba et al. (2005) for further details). Hereafter when referring to the Vesta family we mean a family only determined by dynamical arguments. also show a V-type spectrum. Today, $31 \mathrm{~V}$-type asteroids that are not member of the family are known in the inner belt (Fig. 1, see Table 4 for the asteroid identification, and Footnote 1 for a definition of velocities at infinity $)^{3}$. Also, Roig \& Gil-Hutton (2006), used photometric data from SLOAN, to identify

${ }^{3}$ Given a difference in proper elements $\delta a, \delta e$, and $\delta i$, the tangential $\left(V_{\mathrm{T}}\right)$, radial $\left(V_{\mathrm{R}}\right)$, and perpendicular (to the orbital plane $)\left(V_{\mathrm{W}}\right)$ components of the velocity at infinity may be computed by inverting the following equations:

$$
\begin{aligned}
& \frac{\delta a}{a}=\frac{2}{n a\left(1-e^{2}\right)^{1 / 2}}\left[(1+e \cos f) \delta V_{\mathrm{T}}+(e \sin f) \delta V_{\mathrm{R}}\right] \\
& \delta e=\frac{\left(1-e^{2}\right)^{1 / 2}}{n a}\left[\frac{e+2 \cos f+e \cos ^{2} f}{1+e \cos f} \delta V_{\mathrm{T}}+(\sin f) \delta V_{\mathrm{R}}\right] \\
& \delta i=\frac{\left(1-e^{2}\right)^{1 / 2}}{n a} \frac{\cos (\omega+f)}{1+e \cos f} \delta V_{\mathrm{W}}
\end{aligned}
$$

where $f$ and $\omega$ are the true anomaly and argument of pericenter at the instant of impact (we used $f=75^{\circ}$ and $\omega+f=60^{\circ}$ in Fig. 1B) and C). The velocity at infinity is defined as the quadratic sum of $V_{R}, V_{T}$, 
254 asteroids that are compatible with a V spectral classification. Of these objects, 224 are in the region of the Vesta family (we define this region as the zone between 2.2 and $2.49 \mathrm{AU}$ in semimajor axis, 0.065 and 0.19 in eccentricity, and $3.0^{\circ}$ and $8.25^{\circ}$ in inclination) and are not members of the family (see Fig. 1). While the SLOAN data does not allow a precise spectral identification, these 224 objects may be considered good candidates for spectral identification. Hereafter in this work we refer to these objects as SLOAN candidates. Most of these asteroids have orbits corresponding to ejection velocities with respect to (4) Vesta that are larger than $1 \mathrm{~km} \mathrm{~s}^{-1}$, which is the maximum possible ejection velocity supposed to be produced in the cratering event that formed the Vesta family (Asphaug 1997). Moreover, most of these objects have orbits that differ from those of current Vesta family members because of their proper $e$ and $i$, not because of their $a$. A simple diffusion via Yarkovsky effect, which mostly changes the asteroid semimajor axis, could not explain their current orbital configuration.

In Carruba et al. (2005) we investigated the possibility that asteroids migrated due to the combined effect of chaotic diffusion in nonlinear secular resonances and the Yarkovsky effect, and we showed how this mechanism could explain the current orbital location of (956) Elisa and (809) Lundia. In this article we study another possible mechanism of dynamical migration: close encounters of family members with (4) Vesta itself.

In Sect. 2 we discuss a new symplectic integrator that was developed to account for both the Yarkovsky effect and close encounters with massive asteroids. In Sect. 3 we show the results of a 500 Myr simulation of a fictitious Vesta family and how a model based on the probability density function of having an encounter with (4) Vesta can accurately reproduce the results of the numerical simulation in one dimension (reader interested in how the model was derived may find it useful to read Appendix B); in Sect. 4 we extend our model to three-dimensions. In Sect. 5 we estimate the diffusion probabilities due to repeated close encounters with (4) Vesta at the current location of the known Vtype asteroids outside the Vesta family. We used the results of our model to set constraints on the Vesta family age and initial ejection velocity field. Finally, in Sect. 6 we present our conclusions.

\section{Methods: effects of close encounters with (4) Vesta and the Yarkovsky effect}

To investigate the long-term relevance of close encounters, together with the Yarkovsky effect, we need a numerical tool able to simulate both effects simultaneously. Until now, there were either codes able to simulate close encounters between a massive planet and massless particles (SWIFT-SKEEL, SKEEL hereafter, Levison \& Duncan 2000) or codes to simulate the dynamical diffusion due to the Yarkovsky effect (SWIFT-RMVSY, RMVSY hereafter, Brož 1999). In this work we developed a new symplectic integrator, the SP integrator, based on the Skeel-Biesiadecki scheme of Hamiltonian splitting

and $V_{\mathrm{W}}$. Ejection velocities are given by the quadratic sum of velocities at infinity and escape velocities, where escapes velocities are given by:

$V_{\mathrm{esc}}=\sqrt{\frac{2 G M}{R_{V}}}$

with $R$ the radius of the parent body, $M$ the original mass, and $G$ the gravitational constant.
(Biesiadecki \& Skeel 1993), which also accounts for both the diurnal and seasonal versions of the Yarkovsky effect ${ }^{4}$.

To explain how the integrator was developed we need to discuss some characteristics of both RMVSY and SKEEL. SKEEL uses heliocentric canonical variables (or democratic heliocentric) and splits the Hamiltonian in three parts: i) a Keplerian part, which is advanced with the Gauss $f$ and $g$ functions (Danby 1988); ii) a perturbation that only depends on the heliocentric coordinates and produces a kick in the barycentric momenta, and iii) a third part related to the baricentric motion of the Sun, which causes a linear drift in the heliocentric coordinates (which in the numerical integrator SyMBA (based on the SKEEL algorithm) may be incorporated into the Keplerian part). The equations corresponding to these parts can be rewritten into the heliocentric coordinates and velocities. The third part of the acceleration is evaluated by letting the coordinates drift along a straight line. For a more in-depth description of SKEEL, we send the reader to Levison \& Duncan (2000).

RMVSY instead uses Jacobi variables. In these variables, the Hamiltonian can be split into two parts: a Keplerian part solved using the Gauss $f$ and $g$ functions and a part that is only function of the Jacobian coordinates and that may be expanded in terms of the heliocentric coordinates and integrated by pieces with the classical kick-drift-kick scheme of the leap-frog integrators. A much more detailed explanation of the code can be found in the Brož thesis (1999); here we just point out that we used Vokrouhlický model (Vokrouhlický 1999) for the diurnal and seasonal version of the Yarkovsky effect, so that the drift rate in semimajor axis is given by:

$\frac{\mathrm{d} a}{\mathrm{~d} t}=k_{1} \cos \epsilon+k_{2} \sin ^{2} \epsilon$

where $k_{1}$ and $k_{2}$ are functions depending on the surface thermal parameters and the size, and $\epsilon$ is the spin axis obliquity (see Vokrouhlický 1999 for the exact expression of the $k_{1}$ and $k_{2}$ functions). To compute the Yarkovsky acceleration in SKEEL, we first converted the accelerations from the barycentric into the heliocentric frame. We then added the third piece of the acceleration (axh0, ayh0, and azh0, computed by the subroutine getacch_ah0.f in RMVSY) and computed the Yarkovsky drift. Finally, the linear accelerations were subtracted and the accelerations were then converted back into the barycentric frame.

To test the new code, we simulated the evolution of 25 asteroids (treated as test particles) belonging to the Gefion family. We chose the Gefion family because our previous work on gravitational diffusion by massive asteroids showed us that it is one of the families most affected by this effect (Carruba et al., 2003). We performed two sets of simulations. For the first set, we assumed asteroids with large sizes $(D>65 \mathrm{~km})$ so as to make the Yarkovsky effect negligible. This set was integrated over 100 Myr using SKEEL code and our new SP code. The results are shown in Figs. 2A, C, respectively. For the second set, we assumed the asteroids are very small $(D=15 \mathrm{~m})$ so as to obtain a substantial Yarkovsky drift. This second set was integrated over 10 Myr with the RMVSY and SP codes, and the results are shown in Figs. 2B, D, respectively. In both sets, we included the three most massive asteroids (Ceres, Pallas, and Vesta) as perturbing bodies besides the planets. In the simulations with the RMVSY and SP codes, we used the following physical parameters to compute the Yarkovsky accelerations: bulk density $\rho_{\text {bulk }}=2500 \mathrm{~kg} / \mathrm{m}^{3}$ (this is the density of the asteroid as a

4 We called the new integrator SP, in honor of São Paulo, the city where, at some point in their lives, all authors of this article have lived. 

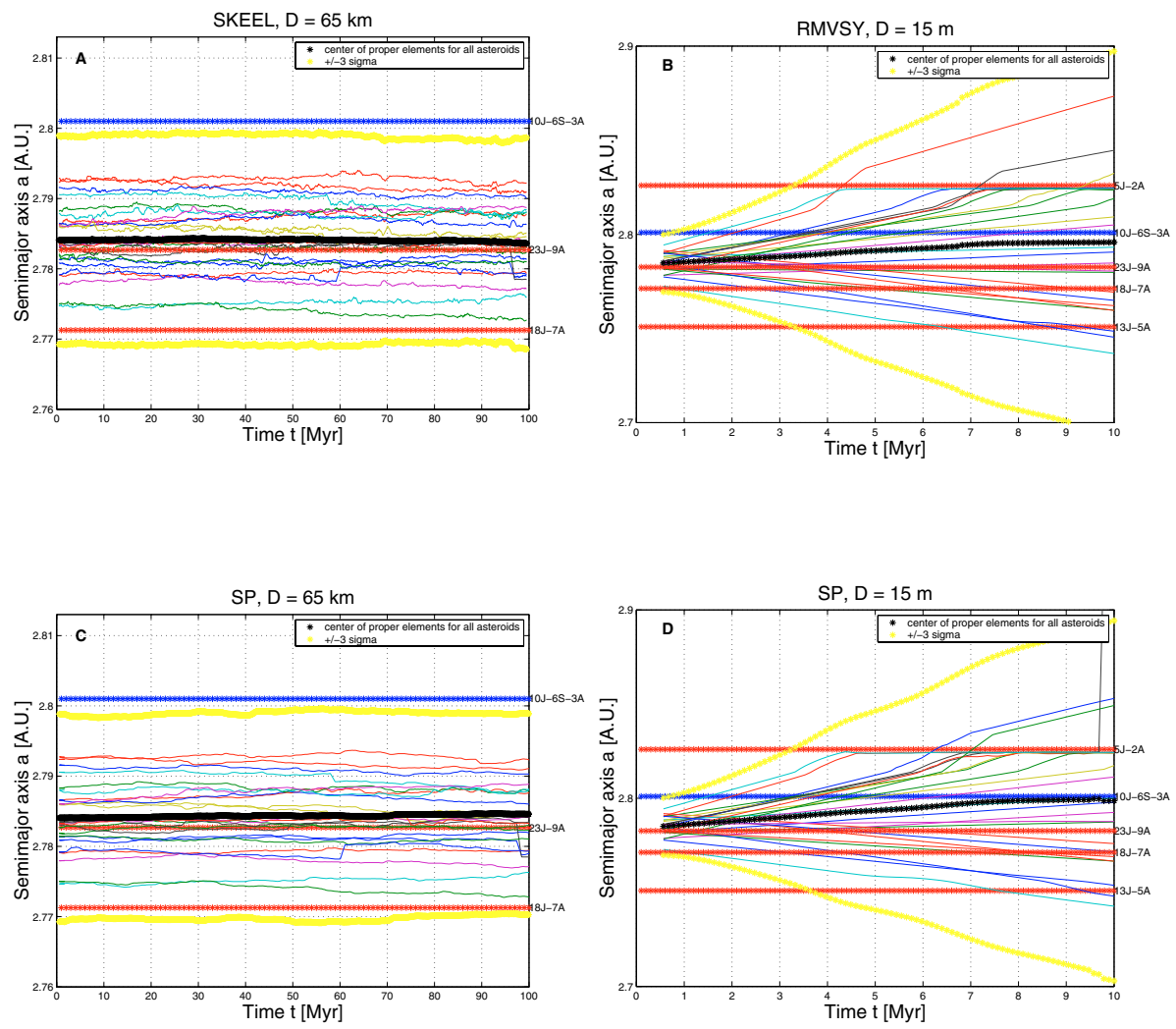

Fig. 2. Time evolution of proper semimajor axes of test particles in the region of the Gefion family for a simulation involving A) SKEEL and large particles $(D=65 \mathrm{~km})$, B) RMVSY and $15 \mathrm{~m}$ diameter particles, C) the new code (SP) and $D=65 \mathrm{~km}$ test particles, and D) SP and $d=15 \mathrm{~m}$ particles. The black line represents the center of the family, the yellow lines a three-sigma level, the red lines show the location of two-body mean-motion resonances, and the blue lines the location of three-body mean-motion resonances. For the definitions of mean-motion and secular resonances, please see Appendix A. whole, i.e., just the ratio of the asteroid mass to its volume), surface density $\rho_{\text {surface }}=1500 \mathrm{~kg} / \mathrm{m}^{3}$ (by this we mean the density of the material on the asteroid surface), thermal conductivity $K=0.001 \mathrm{~W} / \mathrm{m}^{3} / \mathrm{K}$, thermal capacity $C=680 \mathrm{~J} / \mathrm{kg} / \mathrm{K}$, Bond Albedo $A=0.1$, and thermal emissivity $\epsilon=0.9$. We also used random orbital periods and did not reorient the asteroid spins during the simulations.

If the SP code works properly, differences between Figs. 2A, C should be minimal and due to the almost negligible Yarkovsky effect simulated by SP. Differences between Figs. 2B, D should also be minimal and due to (i) a better treatment of close encounters with massive asteroids by the SP code, and (ii) interactions of the test particles with strong mean motion resonances. In fact, we checked the behavior of all 25 orbits in Figs. 2B, D and the remaining orbits show the same time behavior (with the exception of three orbits that in the integration with RMVSY jumped through the $5 / 2$ resonance while in the integration with SP is captured in this resonance (or viceversa)).

To more accurately test the results of the new SP code, we computed the fractional change in orbital elements between one simulation and the other. For example, for the first set of simulations we computed the quantity $\left(a_{\mathrm{SKEEL}}-a_{\mathrm{SP}}\right) / a_{\mathrm{SKEEL}}$ where $a_{\text {SKEEL }}$ and $a_{\mathrm{SP}}$ are the semi-major axes from the integrations with SKEEL and SP, respectively (and so forth for the other orbital elements). We computed mean elements using a runningbox technique (i.e., we computed the mean over a box covering $1 \mathrm{Myr}$, and then shifted our box by 100000 years, and repeated the process until reaching the end of our simulation. We then determined the mean and standard deviations of these fractional changes over all the 25 orbits of the set. Finally, we compared these quantities to the numerical error of the SKEEL code. An estimate of this numerical error was obtained by computing the mean and standard deviations of the fractional changes in orbital elements over the same 25 orbits integrated with SKEEL twice over 100 Myr. We verified that the mean values of fractional changes in orbital elements was less than the mean value due to numerical errors to within three standard deviations. A similar result was obtained when comparing the second set of simulations with RMVSY and SP.

The final step was to compare the result of a simulation with the new code with and without massive asteroids. We did a simulation with asteroids with $2 \mathrm{~km}$ diameters, with and without the three most massive asteroids in the main belt. Results show that the code is able to account for the effect of close encounters, as already shown in the previous simulations. Based on these results, we believe the new code works correctly.

\section{Close encounters with (4) Vesta}

Now that we have a numerical tool for simulating close encounters with (4) Vesta and the Yarkovsky effect simultaneously, we can try to apply it to the case of the Vesta family, in order to determine if close encounters with Vesta can generate a diffusion in proper $e$ and $i$ that is large enough to explain the current population of V-type asteroids outside the family. To better estimate the rates of diffusion in $e$ and $i$ due to close encounters, we generated a fictitious family (of 95 members) and integrated their orbits with the new code, under the action of the planets from Venus to Neptune, plus (4) Vesta.

We used the approach described in Carruba et al. (2003) to obtain the initial orbital distribution $\left(f_{\mathrm{KE}}=0.01\right.$, where $f_{\mathrm{KE}}$ is a factor that takes into account that only a portion of the specific impact energy goes into the fragment's kinetic energy). The sizes were chosen to simulate the size distribution of the V-type asteroids outside the Vesta family. They range from $6 \mathrm{Km}$ to $800 \mathrm{~m}$ in radius, using the albedo of (4) Vesta. We used the following thermal parameters: bulk density $\rho_{\text {bulk }}=3500 \mathrm{~kg} / \mathrm{m}^{3}$, surface density $\rho_{\text {surface }}=1500 \mathrm{~kg} / \mathrm{m}^{3}$, thermal conductivity 
Table 1. The physical (radius and mass) and osculating orbital parameters of (4) Vesta, plus the mean anomaly at the moment of impact used to generate the fictitious Vesta family. The physical parameters were taken from the Horizons website of the Jet Propulsion Laboratory (http://ssd.jpl.nasa.gov/horizons.cgi).

\begin{tabular}{ccccccccc}
\hline \hline$R[\mathrm{~km}]$ & Mass [kg] & $a$ [AU] & $e$ & $i$ [degrees] & $\Omega$ [degrees] & $\omega$ [degrees] & $M$ [degrees] & $M_{\text {imp }}[$ degrees] \\
\hline 234.15 & $3.361 \times 10^{20}$ & 2.36075366 & 0.08974047 & 5.55347431 & 103.086777 & 150.527315 & 340.545548 & 75 \\
\hline
\end{tabular}
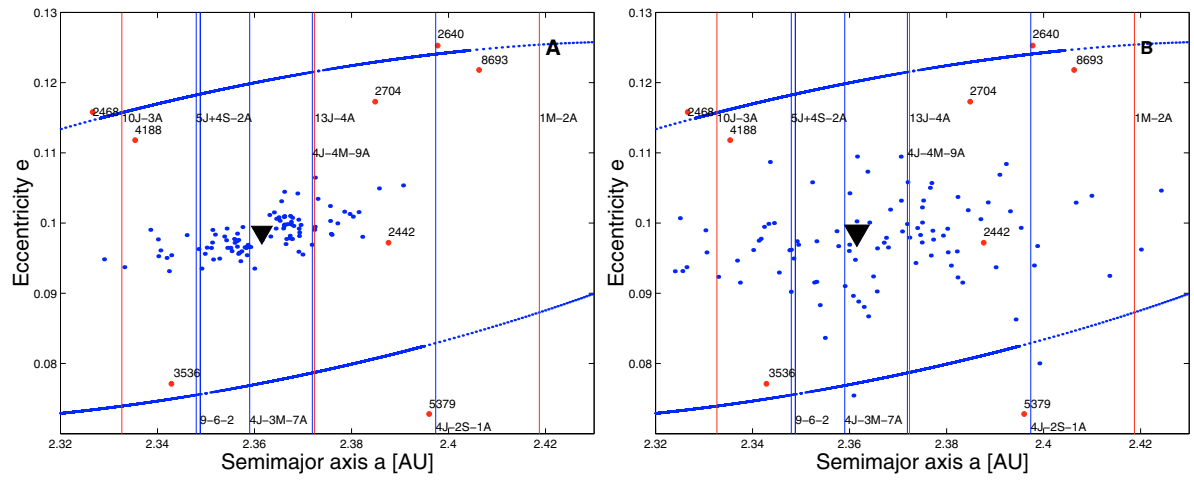

Fig. 3. Initial A) and final $(t=500 \mathrm{Myr})$ B) distribution in the proper $a-e$ plane of the elements of the members of the fictitious Vesta family. The ellipse represents the line of $450 \mathrm{~m} \mathrm{~s}^{-1}$ of maximal initial velocity at infinity. Two-body mean-motion resonances are identified by vertical red lines, and three-body mean-motion resonances are shown as blue lines.
$K=0.001 \mathrm{~W} / \mathrm{m}^{3} / \mathrm{K}$, thermal capacity $C=680 \mathrm{~J} / \mathrm{kg} / \mathrm{K}$, Bond albedo $A=0.1$, and thermal emissivity $\epsilon=0.9$ (Farinella et al. 1998). The angular variables longitude of the node $\Omega$, argument of pericenter $\omega$, and mean anomaly $M$ of the family members were those of (4) Vesta. Table 1 reports the physical and orbital parameters of (4) Vesta, with the values of $\omega$ and $f$ used for generating the fictitious family.

We consider maximum drift, i.e., no reoerientations, no YORP effect, and $0^{\circ}$ or $180^{\circ}$ obliquity (both states have an equal probability of being assigned in our initial conditions), and the thermal parameters used for generating the fictitious family. The rotation rates were assigned by assuming they followed a Gaussian distribution peaked at $\simeq 6 \mathrm{~h}$ and truncated for periods shorter than $2 \mathrm{~h}$ and longer than $12 \mathrm{~h}$.

In our simulations, a particle suffered a close encounter if its distance from (4) Vesta or one of the other massive asteroid was less than the Hill's radius of the massive asteroid, defined as:

$R_{\text {Hill }}=a\left(\frac{M_{A}}{3 M_{*}}\right)^{\frac{1}{3}}$,

where $a$ is the massive asteroid semimajor axis, $M_{A}$ the mass of the asteroid, and $M_{*}$ the mass of the Sun $^{5}$. In our simulations, particles that experienced an encounter occurring at $r_{\text {min }}<R_{\text {Vesta }}$ (with $R_{\text {Vesta }}$ the Vesta radius) were removed from the simulation.

We refer to results obtained for members of the fictitious Vesta family, over 500 Myr. Figure 3 shows the initial and final distribution of the simulated test particles in proper $a-e$ elements. We computed the proper elements with the procedure already described in Carruba et al. (2005) (analogous to synthetic proper elements of Knežević \& Milani 2000). From our results, we computed the time evolution of mean values of changes in $a, e$, and $i$ only due to close encounters for all simulated particles, and the corresponding standard deviations. This was done

5 There are other means to track whether a close encounter occur, such as, checking if the mutual distance was less than the radius of the activity sphere, defined as $r_{\text {act }}=a\left(\frac{m}{M}\right)^{2 / 5}$. Other criteria gave a distance that is generally less than the Hill's radius, so we preferred to use the Hill's radius criteria. It must be pointed out that encounters that occur at large distances and relative velocities only cause small changes in proper elements and are not extremely important in affecting the distribution of proper elements for the family as a whole (Carruba et al. 2003). We therefore believe that the use of our criterion is justified.
Table 2. Drift rates (and relative errors) in proper $a, e, i$ due to close encounters with massive asteroids per $100 \mathrm{Myr}$.

\begin{tabular}{ccc}
\hline \hline$\Delta a$ & $\Delta e$ & $\Delta i$ \\
$\mathrm{AU} /(100 \mathrm{Myr})$ & $1 /(100 \mathrm{Myr})$ & degrees $/(100 \mathrm{Myr})$ \\
\hline$\left(2.0_{-2.0}^{+2.5}\right) \times 10^{-3}$ & $(2.0 \pm 2.) \times 10^{-3}$ & $(6 \pm 5) \times 10^{-2}$ \\
\hline
\end{tabular}

by computing the variations in $a, e$, and $i$ due to each close encounter with a massive asteroid for each particle, and by determining the average and standard deviation of these variations for all the simulated asteroids (Table 2).

We found a drift rate in $a$ of $\left(2.0_{-2.0}^{+2.5}\right) \times 10^{-3} \mathrm{AU} /(100 \mathrm{Myr})$. While the errors do not allow a conclusion, the average value is twice the drift rate found by Carruba et al. (2003) for members of the Gefion family. This may be due to the fact that we integrated test particles extremely close in phase space to (4) Vesta, with a high initial collision probability. As the particles diffused to regions of lower collision probability, we observed that the drift rates due to close encounters diminished as well. The values of drift rates we obtained may therefore be intended as maximal estimates and cannot be safely extrapolated beyond two or three times the simulation time span (500 Myr).

In order to extrapolate our results to later times we followed a different approach from the one used in Nesvorný et al. (2002), and in Carruba et al. (2003). In those papers the authors computed the standard deviation of changes in proper elements and fitted it with a power law of the form

$\sigma=$ Const. $\cdot t^{H}$

where $H$ (in the case of fractional Brownian motion) is the Hurst exponent, which is a measure of how much each step in the diffusion process does or does not depend on previous steps. For $H=0.5$, the motion is uncorrelated and we have the typical one-dimensional Brownian motion. For $H>0.5$ the process is said to be correlated and persistent: each data value is related to some number of preceding values. Values of $\mathrm{H}$ in the range $0,0.5$ are for data series that are anti-persistent: each data value is more likely to have a negative correlation with preceding values.

A problem when using Eq. (4) is that with this approach we may only extrapolate the behavior of a single moment of the distribution, with a considerable loss of information on the 
distribution itself. In this work, therefore, we prefer to develop a different method.

\subsection{Defining the probability density function}

When dealing with diffusion of asteroids via close encounters with massive asteroids, it is useful to define the number density of asteroids per given proper element. Having a distribution of values of $x$ (where $x$ can be the proper $a, e$, or $i$ ), then the number of asteroid in a given interval $x$ and $x+\Delta x(N(x))$ can be derived by integrating the number density $C(x)$ over that interval (where $C(x)$ is defined so that $N(x)=\int_{x}^{x+\Delta x} C(x) \mathrm{d} x$. This number density is not fixed in time, but varies due to the diffusion produced by close encounters. Given the number density of asteroids in one of the proper element $x$ at time $t$, the density at time $t+T$ may be determined via (Hughes $1995^{6}$ ):

$C(x, t+T)=\int \psi\left(x^{\prime}-x, T\right) C\left(x^{\prime}, t\right) \mathrm{d} x^{\prime}$,

where $\psi$ is the so-called probability density function, with dimension $1 / x$, whose integral in the range $x, x^{\prime}$ gives the probability of changing the element $x$ by $\Delta x=x^{\prime}-x$ over time $T$ ( $p d f$ hereafter). Equation (5) can be derived based on the assumption that $x^{\prime}$ and $x$ could be considered as independent random variables (Hughes 1995, pp. 34-35), and it holds on the assumption that the $p d f$ does not change with time (stationary Markovian process of diffusion ${ }^{7}$ ). Later in Sect. 3.2 we will verify this assumption. There are two constrains that Eq. (5) must satisfy. The $p d f$ at any time must be normalized to 1 over the interval of possible values of $x$, or, more generally,

$P(-\infty<x<\infty)=\int_{-\infty}^{\infty} \psi(x) \mathrm{d} x=1$,

and the total number of asteroids $\left(N_{t o t}\right)$ must be conserved over time, i.e.:

$\int_{-\infty}^{\infty} C(x, t+T) \mathrm{d} x=\int_{-\infty}^{\infty} C(x, t) \mathrm{d} x=N_{\text {tot }}$

Knowing the $p d f$, it is therefore possible to propagate the number density of asteroids at later times. To determine the $p d f$, we first computed the changes in proper elements $(\Delta a, \Delta e, \Delta i)$ only caused by close encounters with (4) Vesta (for this we registered in our code every single encounter and computed the proper element before and after the interaction with (4) Vesta). Assuming that, in the limit of large numbers of asteroids, the frequency converges to the probability, the histogram of changes in proper element will give us the $p d f$. Figure 4 shows the histogram of changes in proper $e$ (similar plots were determined for proper $a$ and $i$ ) over 150 Myr obtained by using 11 bins in $\Delta e$ going from -0.011 to 0.011 , in steps of 0.002 ). As already noticed in Carruba et al. (2003), the distribution of changes mostly follows a normal distribution, with the exception of a tail of very close encounters that causes the largest changes in proper elements. The tail

${ }^{6}$ This method was first introduced by Bachelier (1900a,b) in his thesis on stock market speculation.

${ }^{7}$ A stochastic process $\psi_{t}$ is called Markovian if the relationship

$P\left(\psi_{t} \leq x \mid \psi_{t 1}=y_{1}, \psi_{t 2}=y_{2}, \ldots, \psi_{t n}=y_{n}\right)=P\left(\psi_{t} \leq x \mid \psi_{t n}=y_{n}\right)$

holds for all $t_{1}<t_{2}<\ldots<t_{n}<t(n=1,2, \ldots)$ and for all possible values of the random variables considered. $P$ is called stationary distribution if the distributions $P(t, x)$ are equal to $P^{*}(x)$ for all values of $t$, where $P^{*}(x)$ is an initial distribution $P(0, x)$ (Takács 1964).

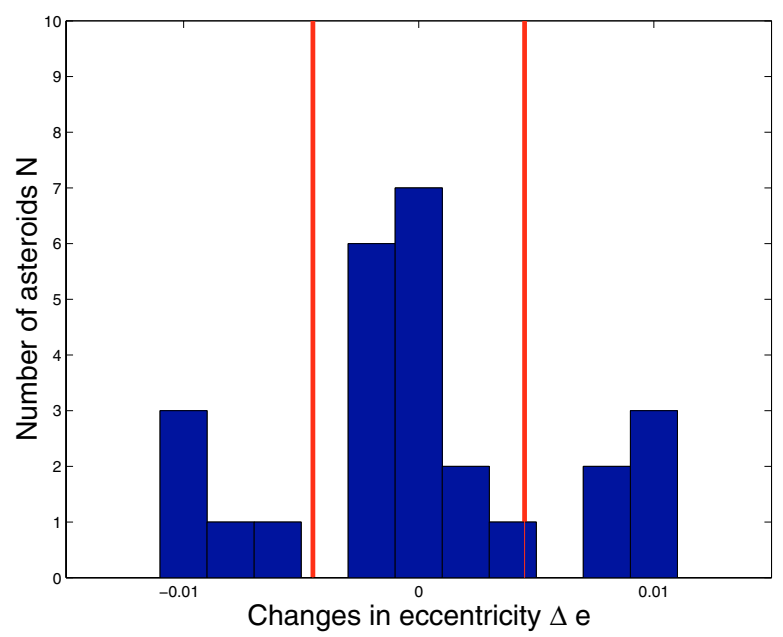

Fig. 4. Histogram of changes in proper $e$ over $T=150 \mathrm{Myr}$ (we do not count the asteroids that were not affected by (4) Vesta in this histogram). The distribution of changes can be divided into two regions: a central region (between the two red lines) that roughly follows a normal distribution and two tails of relative high changes in proper $e$, separated by an intermediate region where very few encounters occurred.

of very close encounters is generally separated from the normal distribution by a region in $e$ where very few encounters occurred. We believe this may be understood if we consider what happens when an asteroid experiences a close encounter with (4) Vesta.

During such an encounter the asteroid will pass at closest approach at a distance $r_{\min }$ with a relative velocity with respect to (4) Vesta $V$. What causes the variation in the asteroid proper elements is not, however, the variation in relative velocity with respect to (4) Vesta but the variation in heliocentric velocity, $\Delta U_{0}$. If we follow Greenberg (1982), the sine of the angle $\chi$ at which the asteroid orbit will be deflected due to the close encounter will be given by:

$$
\begin{aligned}
& \sin \chi=\left[1+\left(\frac{r_{\min } \cdot V^{2}}{G(M+m)}\right)^{2}\right]^{1 / 2}, \\
& \Delta U_{0}=\left(\frac{M}{M+m}\right) V[\sin 2 \chi \boldsymbol{d}+(\cos 2 \chi-1) \boldsymbol{V}]
\end{aligned}
$$

where $\boldsymbol{d}$ is the unit vector pointing from the center of (4) Vesta to the closest approach point and $\boldsymbol{V}$ is the unit vector pointing from the center of (4) Vesta to the direction of the relative velocity. Figure 5A shows a contour plot of changes in heliocentric velocities as a function of $r_{\min }$ and $V$. Note how the encounters at low relative velocities and low minimum distances are the ones that cause the maximum change in heliocentric velocities. Figure $5 \mathrm{~B}$ shows a contour plot of $\Delta U_{0}$ for the region of phase space where encounters at low mutual velocities and distances occur, while Fig. 5C displays an histogram of the same quantity for this region of phase space. Note how the (absolute) change in $\Delta U_{0}$ may be approximated by a region following a normal distribution plus a tail, which ends for a limiting value of $\Delta U_{0}$. The fact that this distribution does not rigorously follow a normal distribution is what makes modeling the long-term effect of close encounter such a difficult task.

We are still confronted with the problem of determining the $p d f$ that can best simulate the results obtained via numerical simulations. The procedure for determining the optimal $p d f$ will be described in the next subsection. 

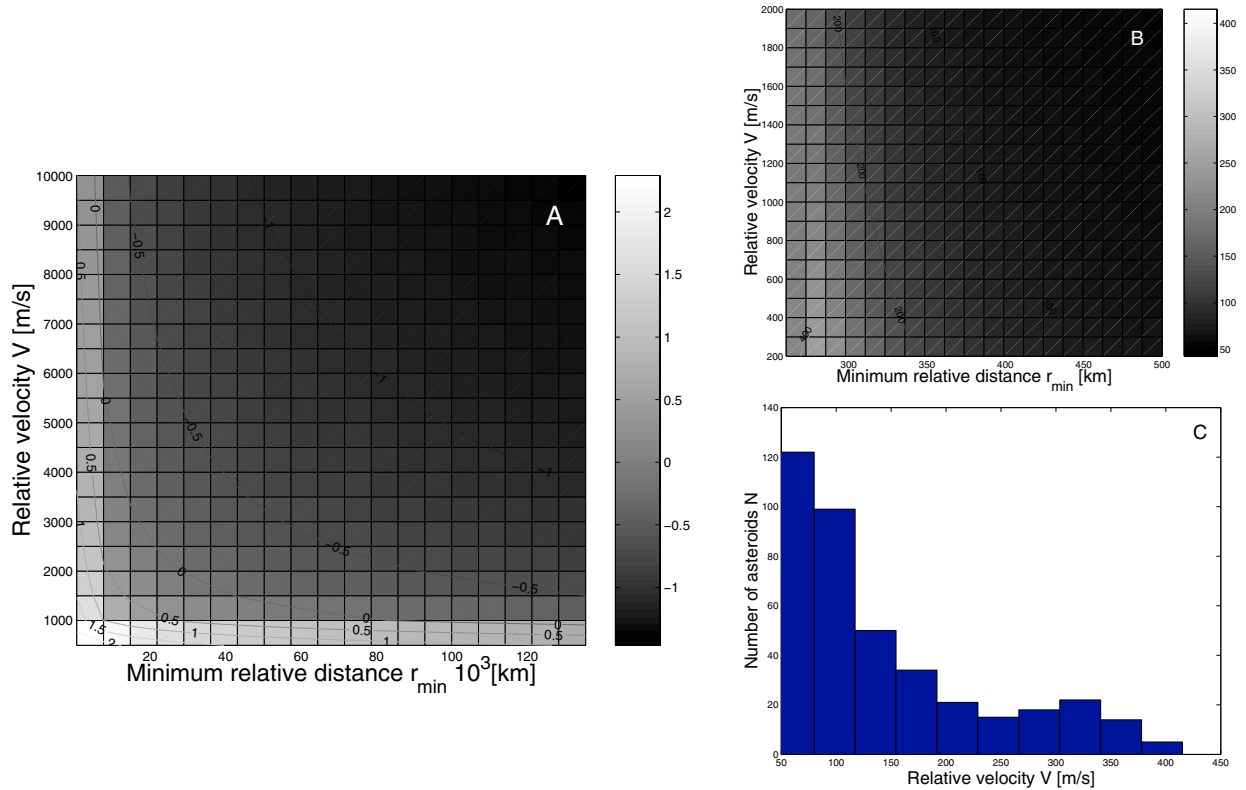

Fig. 5. Contour and color plots of the $\log _{10}$ of changes in heliocentric velocities $\Delta U_{0}$ (in $\mathrm{m} / \mathrm{s}$ ) as a function of $r_{\text {min }}$ and $V, \mathbf{A})$. B) shows a contour and color plot of $\Delta U_{0}$ for the regions where changes of the heliocentric velocities are higher. Finally, C) displays a histogram of the changes of $\Delta U_{0}$ for the same region. Note how the distribution in $\Delta U_{0}$ may be approximated by a Gaussian distribution plus a tail, that corresponds to encounters that occur at low minimum distances and low mutual velocities.

\subsection{Determining the optimal pdf}

As can be seen in Fig. 4 the distribution of changes in proper $e$ is composed of two fairly distinct parts: a central region that roughly follows a normal distribution and two tails. In this work we modeled the $p d f$ by using two distributions: a normal distribution for the encounters that occurred at large minimum distances and relative velocities, and two exponential laws for encounters that occurred closer to (4) Vesta and at lower relative velocities. Other approaches for modeling the $p d f$ can be considered, for instance, we could approximate the tails with quadratic laws, with two linear lines (one going from the normal distribution to the maximum of the distribution in the tails and the other going from the maximum to zero), etc. We prefer to use the exponential law because of its relative simplicity of avoiding the necessity to define another parameter to identify the maximum of the tails' distributions, but other approaches may be worth exploring in future works.

For the case of Fig. 6 and for positive changes in $e$, the exponential part of the $p d f$ was given by

$P(\Delta e)=\exp \left(-\left(s \cdot e_{\min }\right)\right)-\exp (-(s \cdot \Delta e))$,

where $e_{\min }$ is the value of $\Delta e$ where we passed from the Gaussian law to the exponential law, and $s$ is the slope of the exponential. We truncated the distribution at a value of $\Delta e=e_{\max }$. A similar law can be easily derived for negative $\Delta e$. In this model there are several free parameters that need to be adjusted to find the $p d f$ that fits the results of the numerical simulations best. For the Gaussian part we need to determine the standard deviation of the distribution $\sigma$. For the exponential part of the distribution, there are three free parameters: $e_{\min }, e_{\max }, s$. All these parameters must produce a $p d f$ consistent with the observed histograms of changes in $a, e, i^{8}$, and this sets constrains on the possible values of the parameters. For instance, $e_{\max }$ cannot be much larger than the maximum observed change in proper $e$, or we could overestimate the diffusion caused by very close encounters. The value of $e_{\min }$ cannot be much less than the values of $e$ that roughly follow

${ }^{8}$ Since we are fitting distributions in $a, e, i$ that are related to the same set of changes caused by close encounters, we believe our approach to treat the three distributions independently is justified. The validity of this approach will be further discussed in Sect. 4.1.

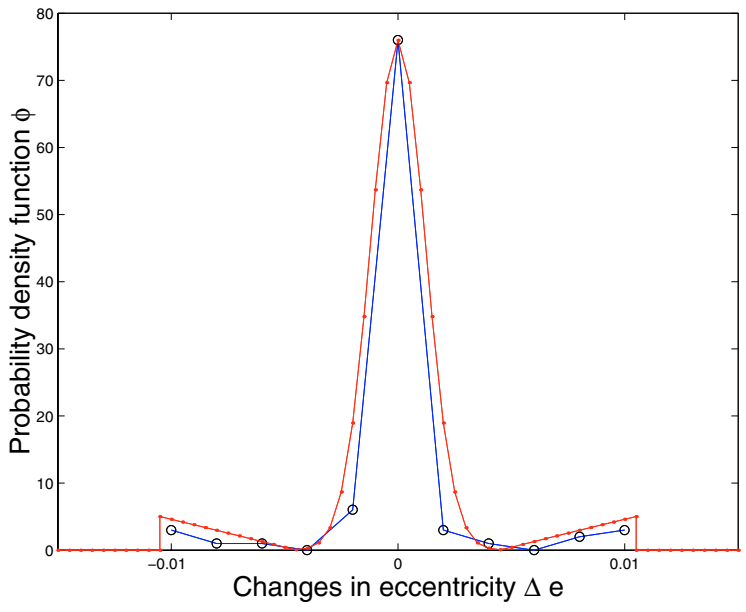

Fig. 6. Histogram of changes in proper $e$ over $T=150 \mathrm{Myr}$ (in blue), with superimposed the $p d f$ that best fit our results (in red).

a normal distribution, $\sigma$ should be comparable to the standard deviation of the changes in $e$ with Gaussian distribution, and $s$ should have a value as close as possible to the slope of the tail parts of distribution in $\Delta e$. Most important, the $p d f$ should be able to reproduce the observed diffusion of the simulated asteroids' proper elements. Finally, we need to determine what the minimum time $T$ is for which Eq. (5) reproduces the distribution observed in the numerical simulation with significant accuracy.

These constrains must all be considered, when a solution for the optimal pdf is sought, and the method must be applied to the distributions in proper $a, e, i$. In Appendix B we describe the method followed to determine the three $p d f \mathrm{~s}$, whose parameters are reported in Table 3. For each of the $p d f$ we verified i) the minimum time $T$ for which Eq. (5) reproduces the distribution observed in the numerical simulation with significant accuracy, and ii) that the process of diffusion may be modeled with a $p d f$ whose shape is independent of time. We refer the reader to Appendix B for more detail. 
Table 3. Values of the parameters $x_{\min }, x_{\max }$ ( $x$ stands for proper $a, e$, and $i), s, \sigma_{\mathrm{el}}$, and the step in element used for the $p d f$ s for proper semimajor axis, eccentricities, and inclination.

\begin{tabular}{cccccc}
\hline \hline & $x_{\min }$ & $x_{\max }$ & $s$ & $\sigma_{\mathrm{el}}$ & step \\
\hline$a$ & $0.003[\mathrm{AU}]$ & $0.011[\mathrm{AU}]$ & 11 & $0.0020[\mathrm{AU}]$ & $0.001[\mathrm{AU}]$ \\
$e$ & 0.0045 & 0.0105 & 12 & 0.0012 & 0.0005 \\
$i$ & $0.15[\mathrm{deg}]$ & $0.40[\mathrm{deg}]$ & 9 & $0.10[\mathrm{deg}]$ & $0.025[\mathrm{deg}]$ \\
\hline
\end{tabular}

\section{Extension to a 3-D space}

Having determined the $1 D-p d f \mathrm{~s}$, we are ready to extend our method to a 3-D space. In this work we assume that the $p d f$ in $a, e, i$ are independent of each other and that integration of the number density of asteroids in $a$ can be done independently from the integration in $e$ and $i$, and vice-versa. We will discuss more the validity of this assumption later in this section.

A more demanding assumption is that the $p d f$ s are constant over the volume of the integration. While this might be acceptable for relatively small regions of space, like the one covered by the asteroids that we simulated, the reality will be more complex for vaster regions. We may think of three cases of $p d f$ : (i) Asteroids that do not cross the orbit of (4) Vesta would have a $p d f$ equal to 0 for any nonzero values of $\Delta a, \Delta e, \Delta i$ (in this case the $p d f$ functions would be a delta function centered in 0); (ii) asteroids that experienced the lowest relative velocity at infinity would have a $p d f$ very similar to the objects we simulated, with a central region following a normal distribution and two sizable tails; (iii) finally, asteroids with a relatively high relative velocity at infinity would be characterized by a $p d f$ following a normal distribution without tails. Understanding how to pass from one case to the other and quantifying the dependence of the $p d f$ on the location in proper element space -so as to obtain a full analytical map of close encounters with (4) Vesta and, possibly, other massive asteroids in the main belt- might be an interesting subject of study. However, this would require extensive numerical simulations of different regions of space, and so would be beyond the scope of this work, which were to pioneer the use of the $p d f$ for modeling the long-term effects of close encounters with massive asteroids. In our opinion, however, before trying to model diffusion of asteroids with different $p d f \mathrm{~s}$, we need to understand how diffusion operates with a single $p d f$, for one and for several bodies, which is the main subject of this section.

Under the assumptions we discussed, the 3-D number density of asteroids at time $t+T$ can be found by a simple triple integral:

$C(a, e, i, t+T)=\int \psi(a) \int \psi(e) \int \psi(i) \cdot C(a, e, i, t) \mathrm{d} a \mathrm{~d} e \mathrm{~d} i,(11$

where $C(a, e, i, t)$ is the number of asteroids per unit $a-e-i$ cell (with a dimension of $1 / \mathrm{AU} /$ degrees). The integration of Eq. (11) must be done by preserving the total number of asteroids $N_{\text {ast }}$, i.e., $\iiint C(a, e, i, t) \mathrm{d} a \mathrm{~d} e \mathrm{~d} i=N_{\text {ast }}$, at all times $t$.

With this constraint we applied Eq. (11) to two sets of initial conditions in proper $a-e-i$ space: the case of an isolated asteroid and the case of the simulated asteroid family. The integration of Eq. (11) was done over a grid in the $a-e-i$ space with steps equal to those used for the respective $p d f$ functions (see Table 3 ) and in the intervals $2.32 \mathrm{AU}<a<2.41 \mathrm{AU}, 0.075<e<$ $0.125,5.0^{\circ}<i<7.5^{\circ}$, so that values of $C$ refer to the number of asteroids per unit cell in the $a-e-i$ space (a single cell has a dimension of $1.25 \times 10^{-7} / \mathrm{AU} /$ degree).

We start our analysis by discussing the case of diffusion of a single asteroid.

\subsection{Diffusion of a single asteroid}

Using Eq. (11) we propagated the proper elements of an asteroid with $a=2.365037 \mathrm{AU}, e=0.099163, i=6.3896^{\circ}$ for $t=t_{\text {in }}+T$, and $t=t_{\text {in }}+2 T$, with $t_{\text {in }}=100 \mathrm{Myr}$ and $T=150 \mathrm{Myr}$.

We start by analyzing the $C$-distribution at $t=t_{\text {in }}+T$. Figure 7 displays isosurfaces of asteroidal number densities for different values of the maximum number density $C$. For $C<1.8 \times 10^{-4}$ we are sampling a region that is accessible to asteroids that experienced encounters in the Gaussian region of the three $p d f$ s (panel I). Asteroids that experienced an encounter that modified one of the proper elements (but not the other two) by a quantity in the exponential region of the $p d f$ can access the volume enclosed by $C<1.2 \times 10^{5}$ (panel II). Asteroids that experienced encounters that modified two elements by quantities in the exponential regions of the $p d f \mathrm{~s}$ can access the volume shown in panel III $\left(C<4.4 \times 10^{-7}\right)$, and, finally, panel IV shows the maximum volume accessible for diffusion in our model $\left(C<10^{-10}\right)$.

We can quantify our qualitative discussion of the distribution of asteroidal number density by introducing diffusion probabilities, which we define as the probability of an asteroid having diffused to regions with values of isosurface number density that is lower than $C_{0}$. This is given by

$P\left(C<C_{0}, t\right)=1-\frac{1}{N_{\text {ast }}} \iiint_{C<C_{0}} C(a, e, i, t) \mathrm{d} a \mathrm{~d} e \mathrm{~d} i$.

For each value of the asteroidal number density $C_{0}$, there is a unique value of $P\left(C<C_{0}, t\right)$. Using Eq. (12 we can compute how the diffusion probabilities depend on $C$ at different times. Figure 8A) shows a semilogarithmic plot of the diffusion probability $P$ as a function of asteroidal number density $C_{0}$. As can be seen in the figure, region I corresponds to a diffusion number density of $67 \%$, region II holds $96 \%$ of the initial population, region III $99.86 \%$, and region IV encloses the total initial population. In our model, the vast majority of asteroids would therefore have experienced at $t=t_{\text {in }}+T$ only encounters in the Gaussian parts of the $p d f$ s or at most an encounter that significantly modified only one of the proper elements. Less than $4 \%$ of the diffused asteroids would have experienced close encounters that modified two of the proper elements significantly and less than $0.14 \%$ would have experienced an encounter that significantly modified the three proper elements at once.

Based on the analysis of these results, we can conclude that the hypothesis of the independence of the three $p d f \mathrm{~s}$ is either correct or that it introduces very small distortions. In our model an asteroid that experienced an encounter that strongly modified, for instance, the proper $a$ could also simultaneously experience strong changes in proper $e$ and $i$ (within the $e_{\max }$ and $i_{\max }$ values of the parameters used to model the $p d f \mathrm{~s}$ ). We believe this is acceptable because: i) in the simulated sample of real asteroid we do observe asteroids that experienced large simultaneous changes in two proper elements; ii) if we compute the velocity necessary to change the proper elements of an asteroid by $a_{\max }, e_{\max }, i_{\max }$ using Gauss' equations (Carruba et al. 2003), the value obtained $(250 \mathrm{~m} / \mathrm{s})$ is inferior to typical values predicted by Greenberg's model (Greenberg 1982) for relative velocities less than $500 \mathrm{~m} / \mathrm{s}$; and iii) even if an encounter that strongly modified the three proper elements at once was not energetically possible, this would only affect less than $0.14 \%$ of the simulated bodies.

Having understood what happens at $t=t_{\text {in }}+T$, we can start to investigate what happens at later times. At $t=t_{\text {in }}+2 T$ we still observe the typologies observed at $t=t_{\text {in }}+T$ (regions I, II, 

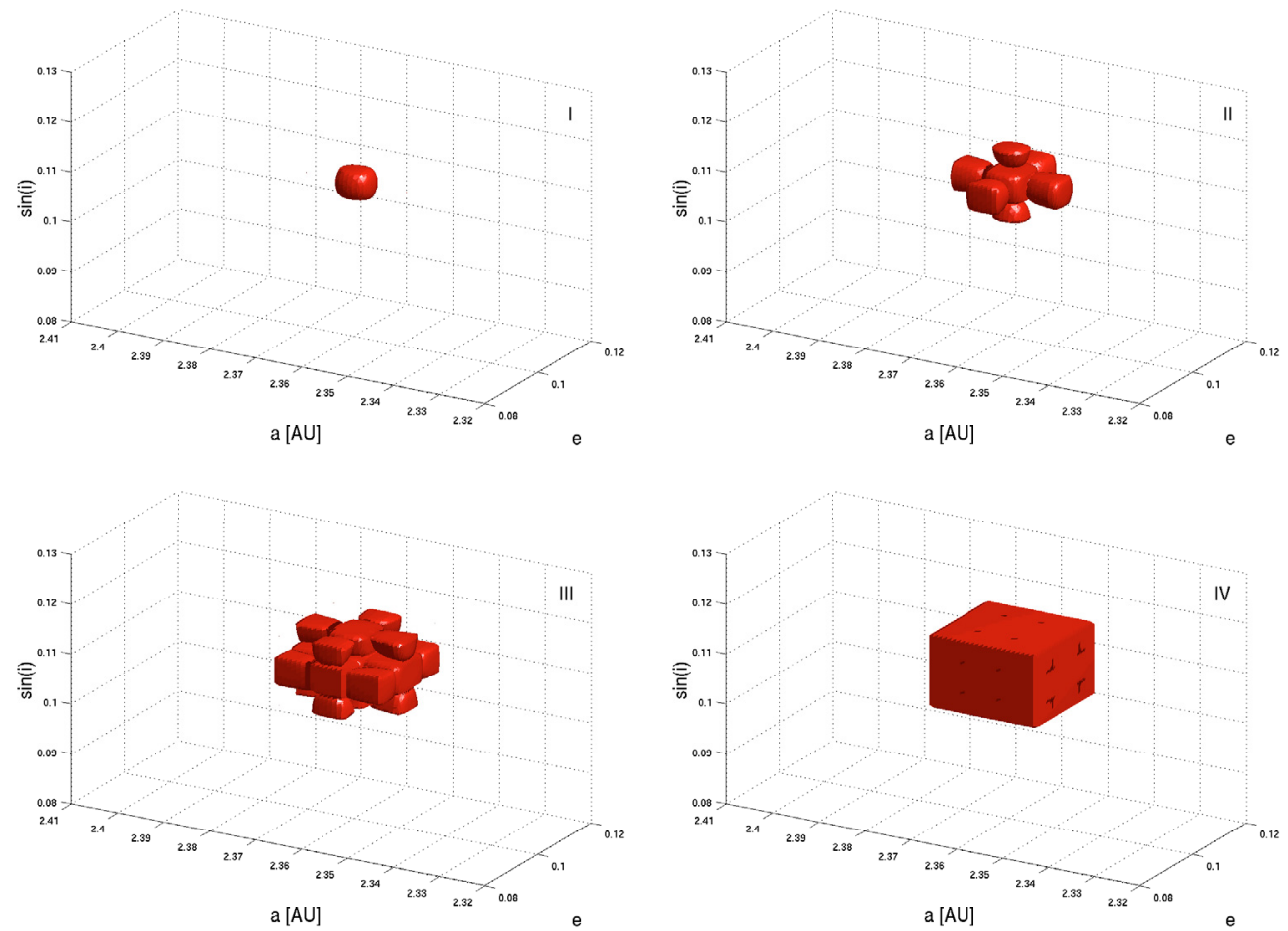

Fig. 7. Isosurface of $C$ in the $a-e-$ $\sin (i)$ space at $t=t_{\text {in }}+T\left(t_{\text {in }}=\right.$ $100 \mathrm{Myr}, T=150 \mathrm{Myr}$ ). The figures (panels I, II, III, and IV) show the four regions of diffusion defined in Sect. 4.1.
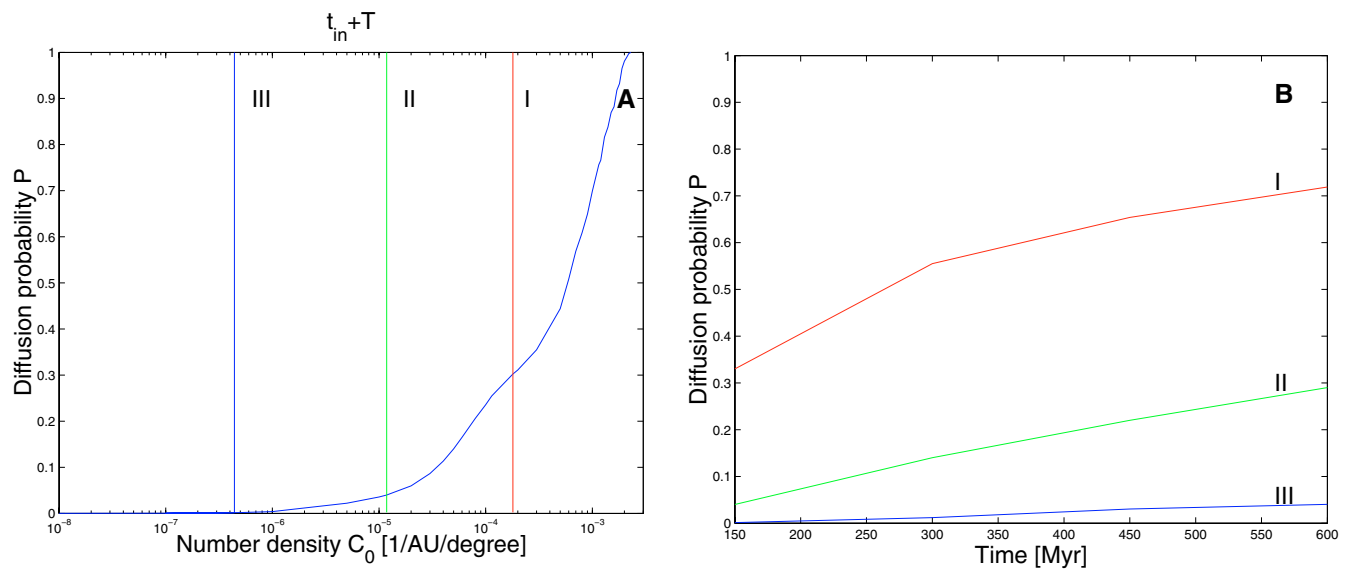

Fig. 8. The asteroid diffusion probability as a function of the $\log _{10}(C)$, at $\left.t=t_{\text {in }}+T \mathbf{A}\right)$. The definition of the four diffusion regions are discussed in Fig. 7. B) shows how the diffusion probability at the limit of the four regions change as a function of time, up to the time for which it is still possible to identify the limits of region III.

III, and IV), plus new typologies that originate from the convolutions of region IV at previous times with the exponential parts of the $p d f$, which results in an increasing number of lobes, until the characteristic parallelepipedal structure seen in Fig. 7D of dimension $2 \cdot x_{\max }$ (where $x$ stands for proper $a, e, i$ ) is reached. A similar behavior, with more complex structures at lower values of $C$, is also observed at later times. We computed how the asteroidal number density $C$ and diffusion probability $P$ at the boundaries of regions I, II, and III vary with time for $t$ going from $T$ to $4 T$. Figure 8B shows these time behaviors: note how, even after four iterations, region III still contains $96 \%$ of the initial asteroidal mass. Also, we observe that, while the limit number density in the Gaussian region (region I) drops with time and the limit number density in region III increases, the limit for region II remains practically constant at $C=1.2 \times 10^{-5}$. In our opinion, the probability levels that separate the four zones at $t=t_{\text {in }}+T$ may be used as a criterion for our extrapolation experiments (see Sect. 5), but, in order to confirm the validity of this criterion, we first need to understand what happens when a whole asteroidal family is propagated via Eq. (11).

\subsection{The diffusion of an asteroid family}

Now that we have seen how the diffusion of a single isolated object operates, we are ready to investigate the diffusion of a whole asteroidal family. We took the proper elements at $t_{\text {in }}=100 \mathrm{Myr}$ of the family we simulated with the SP code as our initial conditions, and diffused the family twice with $T=150 \mathrm{Myr}$. Contrary to the case with a single asteroid where there was essentially expansion in a free space, in the case of a family the diffusion patterns of several asteroids may interact, and, as a result, the asteroidal number density, especially near the family center, may be considerably higher than that created by the diffusion of a single asteroid.

The top panels of Fig. 9 show isosurfaces of $C_{0}=1.2 \times 10^{-5}$ at the two different times, while the bottom panels show contour plots in a plane parallel to the $a-e$ plane passing to the maximum of the number density at the two times. Note how the number densities are significantly higher at the family center than the maximum number densities observed for the single asteroid case. 

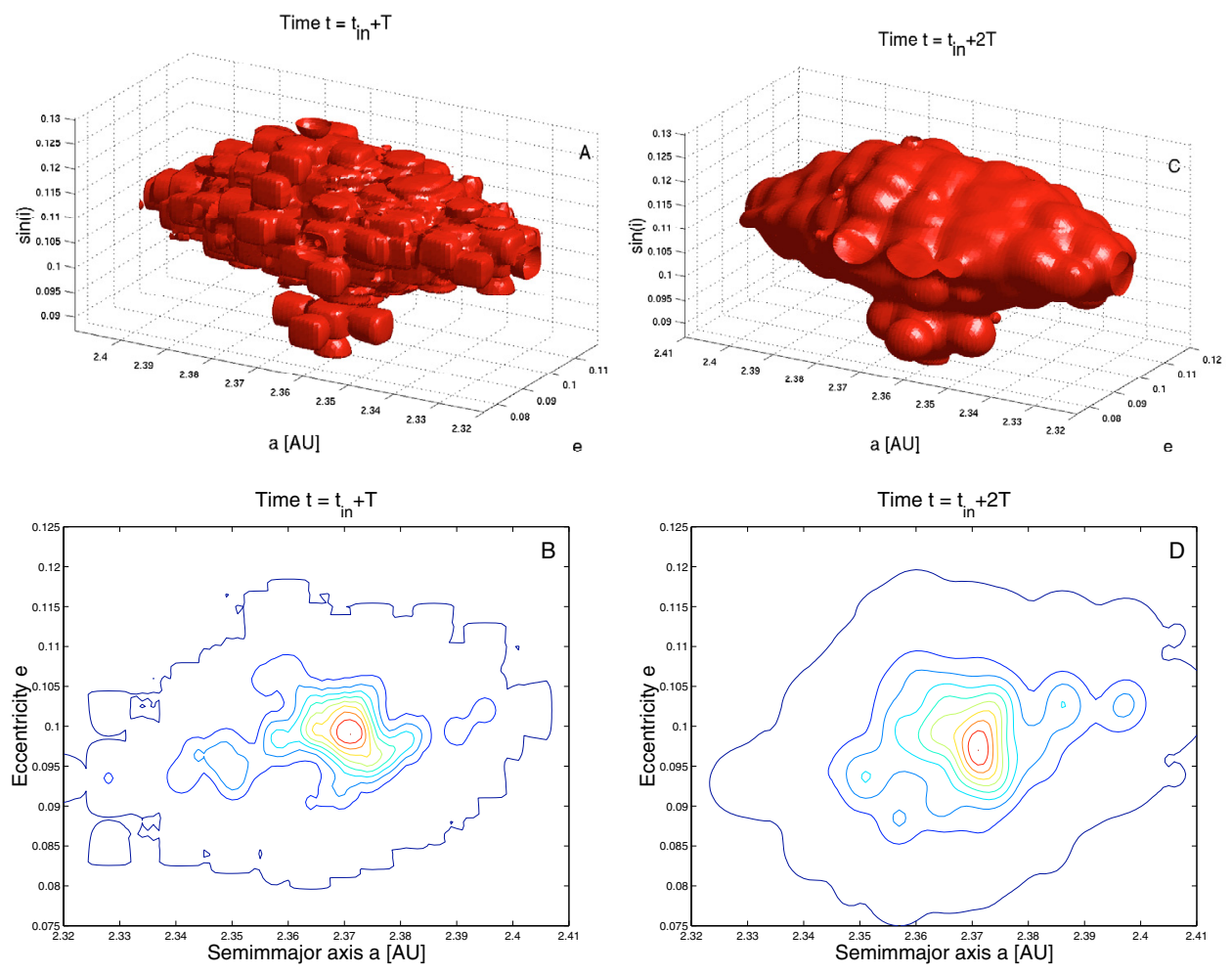

Fig. 9. A) show a 3-D isosurface of the propagated number densities of asteroids at $t=$ $t_{\text {in }}+T=250 \mathrm{Myr}\left(t_{\text {in }}=\right.$ $100 \mathrm{Myr}$ and $T=150 \mathrm{Myr}$ ), for $C_{0}=1.2 \times 10^{-5}$, which enclose $99 \%$ of the propagated asteroids (Eq. (12)). B) shows the $a-e$ contour plot for the $i-$ plane that passes through the maximum number density up to $C=1.2 \times 10^{-5}$ (the limit of region II). C), and D) display the same information, but at $t=t_{\text {in }}+2 T=400 \mathrm{Myr}$.

Another consequence of the addition between asteroidal diffusion patterns is that, while a single asteroid could have experienced a type II or type III diffusion, the value of $C$ at this location may be higher than those allowed for the II and III regions if the asteroid has diffused toward the family center instead of outward. This is exactly what happened in our simulation, where we had two asteroids that experienced a type III encounter during our runs, but diffused inward. Figure 10 shows histograms of $\log _{10}(C)$ at the locations of the simulated asteroids at $t=t_{\text {in }}+T$ and $t=t_{\text {in }}+2 T$. While more than $98 \%$ of the objects remain inside the values of number density associated with the region I for diffusion of a single asteroid, with $2 \%$ of the objects in region II and none in region III and IV, this does not reflect the statistics of encounters, where $10 \%$ of the encounters were of type II and $2 \%$ of type III. We believe this behavior can be explained by the following consideration: in order to reach low values of number densities, an asteroid not only needs to experience a very close encounter, but also needs to be on the family boundary and to diffuse away from the family center. These two additional requirements explain why so few asteroids are found in the II and III regions. However, as the family expands, and the overall asteroidal number density drops, the probability of asteroids on the family boundary experiencing a type II or III encounter going in the right direction increases, and it is therefore more likely to find asteroids at low number densities, as we observe for $t=t_{\text {in }}+2 T$. As time increases and the family expands, the diffusion probabilities at large distances should, in our opinion, asymptotically reach those of a single asteroid.

Based on these considerations, we can set criteria to extrapolate the future behavior of an initial asteroidal number density. In particular, we can define three $C$ values where we expect to find most of the simulated objects. For each iteration, we can use the boundaries of the asteroid region I, II, and III to estimate the diffusion of a real family. We could therefore expect to find at least $67 \%$ of the object in region I, $96 \%$ of the objects in region II, and a few adventurous asteroids in region III, for
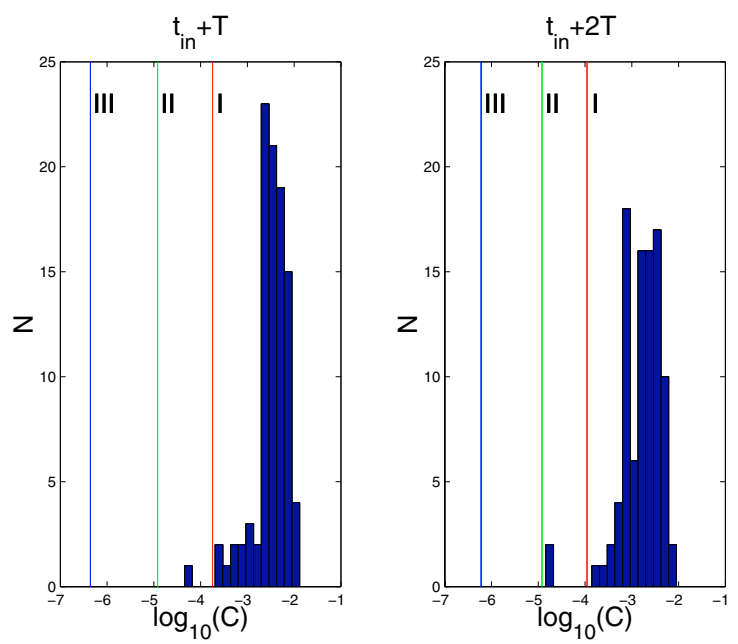

Fig. 10. Histogram of the values of the propagated asteroidal number densities at $t=t_{\text {in }}+T$ (left panel) and $t=t_{\text {in }}+2 T$ (right panel, $t_{\text {in }}=$ $100 \mathrm{Myr}$ and $T=150 \mathrm{Myr}$ ), at the locations of the asteroids simulated with the São Paulo integrator at corresponding times.

$t=t_{\text {in }}+T$, with increasing probabilities of finding objects in regions II and III at later times.

Based on these criteria, we are now ready to start the extrapolation procedure.

\section{Which of the V-type asteroids outside the Vesta family might be former family members scattered by (4) Vesta?}

So far we have used our model to make predictions that were testable on the results of numerical simulations. From now on we are entering the dangerous waters of extrapolation, and we are going to use our model to make predictions on longer timescales 
Table 4. List of the 31 V-type asteroids currently known outside the Vesta dynamical family. We report number, name, proper $a, e$, $i$, absolute magnitude $H$, and the diffusion probability at $t=1200 \mathrm{Myr}$ and $t=2100 \mathrm{Myr}$.

\begin{tabular}{|c|c|c|c|c|c|c|c|}
\hline Ast. \# & Ast. name & $a$ & $e$ & $i$ & $\bar{H}$ & $P(1200)$ & $\overline{P(2100)}$ \\
\hline 809 & Lundia & 2.28311 & 0.1450 & 6.7363 & 12.10 & 0.0 & 0.0 \\
\hline 854 & Frostia & 2.36821 & 0.1635 & 6.4306 & 11.81 & 0.0 & 0.0 \\
\hline 956 & Elisa & 2.29811 & 0.1575 & 6.4076 & 12.03 & 0.0 & 0.0 \\
\hline 1914 & Hartbeespoortdam & 2.40555 & 0.1393 & 4.8473 & 11.96 & 0.01 & 0.33 \\
\hline 1946 & Walraven & 2.29357 & 0.1895 & 7.4349 & 12.09 & 0.0 & 0.0 \\
\hline 2113 & Ehrdni & 2.47377 & 0.0726 & 6.0790 & 12.66 & 0.33 & 1.46 \\
\hline 2442 & Corbett & 2.38765 & 0.0972 & 5.4225 & 12.54 & 58.25 & 87.41 \\
\hline 2468 & Repin & 2.32664 & 0.1158 & 6.4364 & 12.84 & 14.11 & 9.00 \\
\hline 2566 & Kirghizia & 2.45003 & 0.1044 & 4.4219 & 12.20 & 0.0 & 0.20 \\
\hline 2579 & Spartacus & 2.21037 & 0.0807 & 5.8947 & 13.03 & 0.0 & 0.0 \\
\hline 2640 & Hallstrom & 2.39777 & 0.1253 & 6.5286 & 12.85 & 5.28 & 5.52 \\
\hline 2653 & Principia & 2.44350 & 0.1145 & 5.0773 & 12.24 & 1.49 & 9.83 \\
\hline 2704 & Julian Loewe & 2.38485 & 0.1173 & 5.0888 & 12.75 & 3.56 & 14.80 \\
\hline 2763 & Jeans & 2.40355 & 0.1790 & 4.3185 & 11.99 & 0.0 & 0.0 \\
\hline 2795 & Lepage & 2.29574 & 0.0747 & 6.5979 & 12.75 & 1.09 & 2.79 \\
\hline 2851 & Harbin & 2.47819 & 0.1204 & 7.7933 & 11.98 & 0.0 & 0.0 \\
\hline 2912 & Lapalma & 2.28927 & 0.1175 & 6.7421 & 12.45 & 0.13 & 0.28 \\
\hline 3536 & Schleicher & 2.34287 & 0.0771 & 6.6555 & 13.73 & 4.54 & 5.93 \\
\hline 3849 & Incidentia & 2.47422 & 0.0653 & 5.3938 & 12.63 & 0.0 & 0.22 \\
\hline 3850 & Peltier & 2.23457 & 0.1061 & 4.7323 & 13.46 & 0.0 & 0.0 \\
\hline 3869 & Norton & 2.45241 & 0.1009 & 5.2787 & 12.54 & 7.42 & 29.47 \\
\hline 4005 & Dyagilev & 2.45193 & 0.1127 & 5.9984 & 12.67 & 87.80 & 61.95 \\
\hline 4188 & Kitezh & 2.33538 & 0.1118 & 5.6413 & 12.67 & 30.65 & 42.13 \\
\hline 4278 & Harvey & 2.26689 & 0.1473 & 5.2499 & 13.62 & 0.0 & 0.0 \\
\hline 4434 & Nikulin & 2.44110 & 0.1033 & 5.5261 & 12.88 & 38.71 & 69.3 \\
\hline 4796 & Lewis & 2.35523 & 0.1413 & 3.0955 & 13.22 & 0.0 & 0.0 \\
\hline 4977 & Rauthgundis & 2.29229 & 0.0928 & 5.9408 & 13.99 & 49.636 & 43.55 \\
\hline 5379 & Abehiroshi & 2.39599 & 0.0728 & 3.3537 & 12.55 & 0.0 & 0.0 \\
\hline 6406 & 1992 MJ & 2.27549 & 0.1272 & 7.7123 & 13.16 & 0.0 & 0.0 \\
\hline 7148 & Reinholdbien & 2.28587 & 0.0996 & 5.6413 & 13.07 & 24.24 & 31.16 \\
\hline 8693 & Matsuki & 2.40630 & 0.1218 & 6.2692 & 12.65 & 26.79 & 33.19 \\
\hline
\end{tabular}

and different asteroidal distributions than the ones we used in our SP runs. This is a task that must be dealt with while aware that the results are based on several assumptions that may not hold. In the previous subsection we already discussed the problem of the dependence of $p d f$ functions on the location of the asteroids in the $a-e-i$ space. Another assumption that must be taken into account when extrapolating to longer timescales is that the $p d f$ functions remain constant in time. In this work we best-fitted the optimal $p d f$ for two time intervals $T$, and found a robust solution for the $p d f$ s, so as to work under the assumption of a Markovian process of diffusion. Verifying that this hypothesis holds for later times is something that we leave to future research.

With those caveats, we came back to our original problem on the origin of the V-type asteroids outside the Vesta family. Since the publication of our first article on this subject (Carruba et al. 2005), six more asteroids have been identified as V-type in the proximity of the Vesta family (Alvarez-Candal et al., submitted). In Table 4 we report an updated list that includes these new asteroids. We note that according to our numerical simulations the asteroid (6406) $1992 \mathrm{MJ}$ should currently be inside the $z_{2}$ resonance according to our numerical simulations. Our numerical simulations show that the scenario described for the origin of (809) Lundia and (956) Elisa in Carruba et al. (2005) applies to this asteroid as well.

To investigate which asteroids could be explained in the framework of diffusion via close encounters with (4) Vesta, we perform the following experiment: we use as initial conditions the whole Vesta family (5112 members) and propagated the initial asteroidal number densities for $14 T$ (=2100 Myr). We then computed the asteroidal number densities and diffusion probabilities at the locations of the V-type asteroids and of the SLOAN candidates. We need to point out that the diffusion probabilities found in this way are only related to diffusion via close encounters, and ignore other possible mechanisms. For instance, (809) Lundia and (956) Elisa have a zero probability of having reached their current locations only via diffusion by close encounters with (4) Vesta, but from our work of last year (Carruba et al. 2005) we know that the interplay of the Yarkovsky effect with diffusion in nonlinear secular resonance might be responsible for their current orbital positions. Nevertheless, this is a useful exercise because it allows us to identify possible past members that diffused only due to close encounters with (4) Vesta.

Figure 11 displays the locations in the $a-e$ plane of these objects, with different color codes for the bodies that had probability higher than 33\% (limit of zone I, Fig. 11A), 4\% (limit of zone II, Fig. 11B, and $0.14 \%$ (limit of zone III, Fig. 11C of being diffused by (4) Vesta by the end of the simulation. Table 4 reports the diffusion probabilities of the V-type asteroids at $t=1200 \mathrm{Myr}$ (the minimum estimated age of the Vesta family, according to the simulations for (809) Lundia and (956) Elisa, Carruba et al. 2005), and for $t=2100 \mathrm{Myr}$, the end of our simulations.

One interesting piece of information that can be taken from Table 4 is that, while the diffusion probabilities grows with time for most cases, for a few asteroids very close to the family borders ((4977) Rauthgundis, (8693) Matsuki, (2640) Hallstrom) the diffusion probabilities reach a maximum and then drop (see Fig. 12B). This could be explained in terms of the normal diffusion from extended sources. As soon as the majority of the asteroidal population has passed the phase space boundaries of 

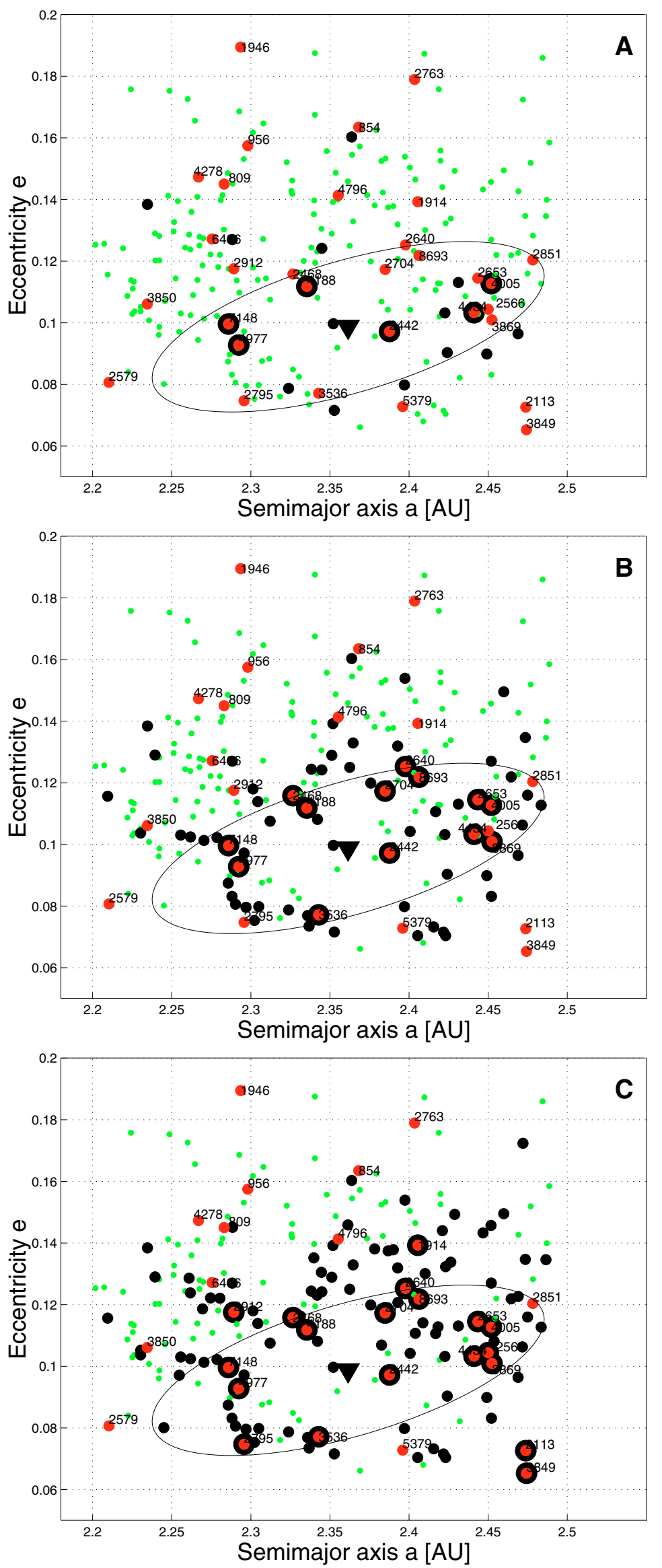

Fig. 11. $a-e$ projection of the V-type asteroids outside the Vesta family (red dots), and the SLOAN candidates (green dots). The V-type asteroids and the SLOAN candidates with a diffusion probability at $t=2100$ Myr higher than $33 \%$ (limit of zone I of diffusion), $4 \%$ (zone II), and $0.14 \%$ (zone III) are identified by larger black dots in $\mathbf{A}$ ), B), and $\mathbf{C}$ ), respectively. The ellipse shows the $450 \mathrm{~m} / \mathrm{s}$ level of maximum ejection velocity, given for reference, and the triangle the orbit of (4) Vesta itself. The fact that some asteroids appear farther away from the family than others that have higher values of diffusion probabilities is an effect caused by the projection on the $a-e$ plane. 

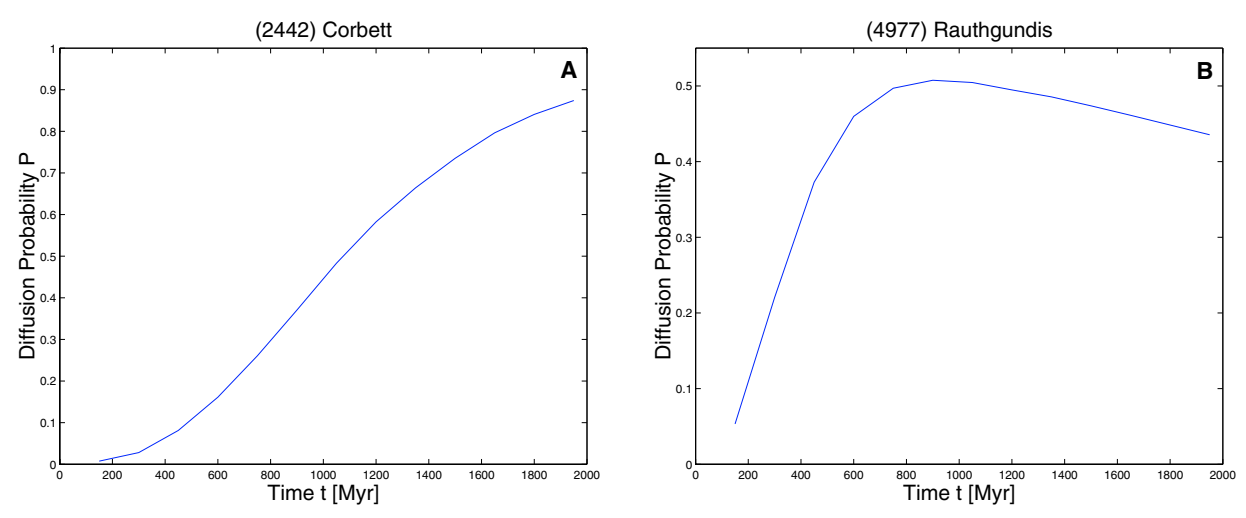

Fig. 12. Diffusion probabilities as a function of time at the location of asteroid (2442) Corbett (left panel) and (4977) Rauthgundis (right panel). In most cases diffusion probabilities slowly grow as a function of time until reaching a maximum (right panel) when most of the asteroids surpass the phase space boundaries of the V-type asteroid. In this case the diffusion probability may actually drop at later times. the V-type asteroid, the diffusion probability starts to decrease. We believe it is interesting to show that for some asteroids the probability of being scattered may actually drop with time!

We are still left with the problem of determining i) which V-type asteroids may be explained in the framework of diffusion via close encounter with (4) Vesta; and ii) how the (unknown) original orbital distribution of the family members affect the diffusion process. We will try to deal with these questions in the next subsections.

\subsection{Dependence on initial ejection velocity field}

The results of Sect. 5 show us that, starting with the current Vesta family members, 18 V-type asteroids outside the Vesta family have a diffusion probability higher than $0.14 \%$ (the limits of region III) at $t=2100$, and can so be interpreted in the framework of diffusion via close encounters only. This is both a conservative estimate, since other mechanisms are at play (Yarkovsky and YORP effect, diffusion in mean-motion and secular resonance or both) that could have modified the proper $a, e$, and $i$ of the asteroids, and a generous estimate, since we started with the current orbital distribution of the Vesta family (the original family most likely had lower values of ejection velocities than the current members).

What we plan to do next is to use the information gathered with this simulation to select those objects that among the 18 candidates were less likely to have interacted with powerful mean-motion or secular resonances and whose current proper $e$ and $i$ was most likely caused by encounters with (4) Vesta. Recently, Vokrouhlický et al. (2006a,b) have used the current proper $a$ distribution of young asteroid families to set constraints on the age, initial velocity field, and on a parameter indicating the strength of the YORP effect, by simulating the diffusion of the original families via the Yarkovsky/YORP effect. Due to limitations in the current understanding of the YORP cycle, this method cannot be applied to families older than $\simeq 1 \mathrm{~Gy}$, like the Vesta family (which is at least $1.2 \mathrm{~Gy}$ based on the minimum time for members of the Vesta family to diffuse to the current orbital locations of (956) Elisa and (809) Lundia et al. 2005).

It is our future intention to see if diffusion via close encounter can play a role in the determination of families' ages. While this is in essence less precise than a determination based on the Yarkovsky/YORP effect, and is probabilistic in nature (it is of course not possible to be certain that an encounter with (4) Vesta did occur in the past), the advantages of such a method would be that i) the proper $e$ and $i$ of selected asteroids could be used to set constraints on the family age and ejection velocities, and not only on the proper $a$; ii) there is in principle no limitation in time for the estimate of the family age; and iii) it does not strongly depend on the details of the YORP cycle. To correctly apply this new methodology, we will need to have a model that includes the Yarkovsky/YORP diffusion with the diffusion due to close encounters with massive asteroids. This exceeds the purposes of this paper and will be dealt with in a future work. Here we prepare the terrain by identifying the best V-type candidates to be explained as former members scattered by (4) Vesta and by starting to study how the diffusion probabilities at the locations of these candidates depend on the initial ejection velocity field.

To do so, we first need to identify asteroids whose proper $e$ and $i$ is mostly due to scattering via close encounters with (4) Vesta. This is not an easy task. Our work on (956) Elisa and (809) Lundia has shown us that diffusion in tiny three-body and secular resonances may play a significant role in the diffusion of the members of families. In another article of the series on the V-type asteroids outside the Vesta family, we are planning to investigate the role that martian nonlinear secular resonances may play. It is rather difficult to find a location in the asteroid belt that is not close to a resonance.

Nonetheless, some of the V-type asteroids outside the Vesta family are indeed in more stable regions than others. Of the 18 candidates we identified, we eliminated those that are close to one of the main secular or mean-motion resonances in the region (see Table 2 in Carruba et al. 2005). We also eliminated objects that were too close to the family boundary and could be actual members, if a higher value of the cut-off used to determine the members of the dynamical family by Mothé-Diniz et al. (2005) is considered. After these considerations, we were left with four promising candidates: (2795) Lepage, (4434) Nikulin, (7148) Reinholdbien, and (8693) Matsuki.

We now need to study how the diffusion probabilities depend on the initial ejection velocity field. We start by noticing that the highest absolute magnitude of the four candidates is 13.07. Of all the Vesta family members, we choose to concentrate on the 82 that have an absolute magnitude lower than 13.10 (except (4) Vesta itself), since smaller objects cannot be identified as the precursors of these V-type objects (this procedure also removes the problem of the uncertainty associated with the family boundary, which depends on the value of the cutoff chosen for the identification via the hierarchical clustering method, since we are only choosing the brightest objects. Tests using the hierarchical clustering method with different velocity cut-offs have confirmed that the $H<13.10$ objects in our family are also found for higher velocity cutoffs, and that, except for cut-offs that are too large, such that the nominal Vesta family would also include known members of nearby families, no significant addition is found to the $H<13.10$ Vesta family population).

Figure 13A show the $a-e$ projection of these objects, superimposed on the $a-e$ of the whole family plus V-type asteroids, 

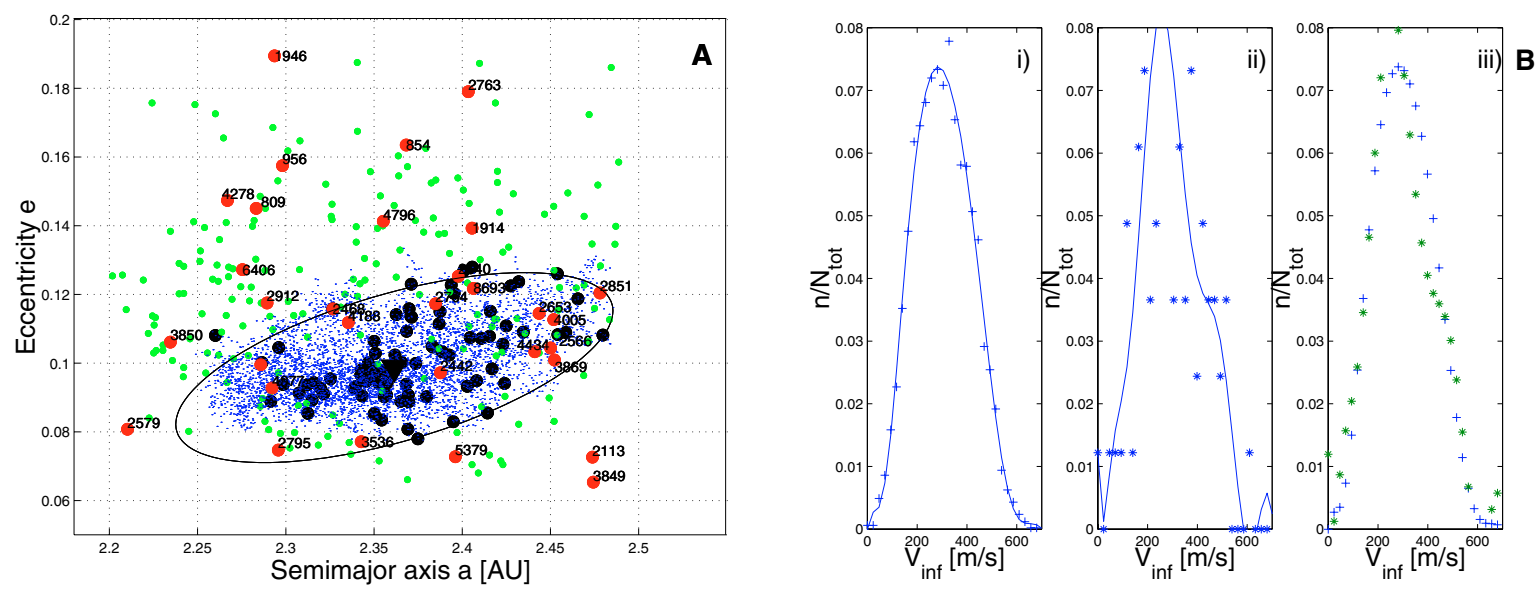

Fig. 13. Left A) the $a-e$ projection of the Vesta family members, V-type asteroids outside the Vesta family, SLOAN candidates, and the family members with absolute magnitude lower than 13.1 (full black dots, for the other objects we follow the same symbolism as in Fig. 1). Note how the asteroids with larger absolute magnitude essentially cover the whole of the Vesta family. On the right $\mathbf{B}$ ), we show histograms of normalized distribution of the velocities at infinity (33 equally-spaced bins in $V_{\text {inf }}$ between 0 and $750 \mathrm{~m} / \mathrm{s}$ ) of i) the whole family; ii) the $H<13.1$ asteroids; and iii) the comparison of the 10th degree polynomial fits to the two distributions (continuous lines in panels i) and ii)). If we consider the errors, the two distributions are in fairly good agreement. This confirms that, rather than been concentrated toward the center of the family, the $H<13.1$ family members' distribution in proper elements is fairly spread out and cover the same range of the family distribution.

while Fig. 13B shows an histogram of the velocities at infinity (computed via Eqs. (5)-(7) in Carruba et al. 2003) of i) the whole family; ii) the $H<13.1$ objects; and iii) the 10th degree polynomial fit to these two distributions. It can be observed that those objects are essentially diffused over the whole family and do not concentrate toward the center. This is an indication that the Vesta family is indeed an old one, since even relatively large bodies have had the time to disperse out to the family boundaries (the 3:1 and the 7:2 mean-motion resonances). We have computed the standard deviation of the tangential, radial, and normal velocity components (with respect to (4) Vesta) of these objects and found values of 305,327 , and $281 \mathrm{~m} / \mathrm{s}$, respectively, with a mean value of $304 \mathrm{~m} / \mathrm{s}$.

To generate the initial distribution of fragments, we follow the same procedure as in Vokrouhlický et al. (2006b), i.e., we assume that all velocity components $V_{\mathrm{R}}, V_{\mathrm{T}}$, and $V_{\mathrm{N}}$ along the radial, transverse, and normal directions with respect to the parent body orbit have the same Gaussian distribution with standard deviation $V_{\mathrm{SD}}$. We also assume that $V_{\mathrm{SD}}=V_{\mathrm{ej}}(5 \mathrm{~km} / D)$ is inversely proportional to the size $D$ and that $V_{\mathrm{ej}}$ is a free parameter, that, in our case, we assume inferior to $304 \mathrm{~m} / \mathrm{s}$. The number of fragments used is the same as the number of observed asteroids in the family with $H<13.1$, with the same absolute magnitude. This is converted to size $D$ using the value of the geometric albedo of (4) Vesta (0.4228), the only object in the Vesta family for which such information is available.

Figure 14 shows the time evolution of the diffusion probabilities at the locations of the four V-type asteroids most likely to be former family members scattered by (4) Vesta, for different values of $V_{\mathrm{ej}}{ }^{9}$. As can be seen in the figure, the time evolution of the diffusion probabilities follows different behaviors in the four cases. For the case of (8693) Matsuki the diffusion probabilities reach the limits of region I, while in other cases, even in the most

9 The fact that, in the cases of (2795) Lepage and (7148) Reinholdbien for some values of $V_{\text {inf }}$, the diffusion probabilities curves intersect at small iteration times as the velocity at infinity increases is caused by the presence of isolated asteroids in the initial conditions generated by our code. As the family as a whole diffuses, the effect of isolated asteroids is smeared out and the curves separate. favorable case of $V_{\mathrm{ej}}>270 \mathrm{~m} / \mathrm{s}, P$ does not reach the limits of region I. If we want to set limits on the Vesta family age based on these experiments, the diffusion process would be dominated by the objects with the lowest diffusion probabilities: assuming that these four asteroids were all produced by diffusion by close encounters with (4) Vesta, the actual age of the family will be dominated by the "slowest" body. In Table 5 we report how the time of the crossing of region III varies as a function of $V_{\mathrm{ej}}$. Our results show that i) the initial ejection velocity field had to be relatively large (at least $210 \mathrm{~m} / \mathrm{s}$, with an estimate of $(240 \pm 60 \mathrm{~m} / \mathrm{s})$ or else (4434) Nikulin, could not diffuse to the current locations in the time covered by the simulations ( $2.1 \mathrm{~Gy})$; and ii) the Vesta family age should be $(1200 \pm 700)$ Myr, so as to be able to produce (4434) Nikulin ${ }^{10}$.

Our first results seem to confirm the work of Asphaug (1997) that predicted that the cratering event that produced the Vesta family should have produced an ejection velocity field characterized by high velocities, while the second estimate agrees with our work of last year, where we predicted a minimum age for the Vesta family of $1.2 \mathrm{~Gy}$ based on the minimum time needed by Vesta family members to reach the current orbital location of (809) Lundia and (956) Elisa via evolution in nonlinear secular resonances forced by the Yarkovsky effect.

Finally, all these considerations neglect the Yarkovsky/ YORP evolution and passages through resonances. While the second assumption seems to be confirmed by the preliminary results of numerical simulations that show that, the effect of passages through the (few) mean-motion resonances in the regions is small for clones of the four asteroids, the first effect should be taken into account. We are planning to develop a more accurate study in a later paper.

\footnotetext{
10 The error on the initial ejection velocity field just reflects the range of velocities for which a solution for all the four asteroids is possible ( $V_{\text {ej }}$ between 180 and $300 \mathrm{~m} / \mathrm{s}$, see Table 5). The error on the family age is the standard deviation of the distribution of possible values of age in Table 5 .
} 


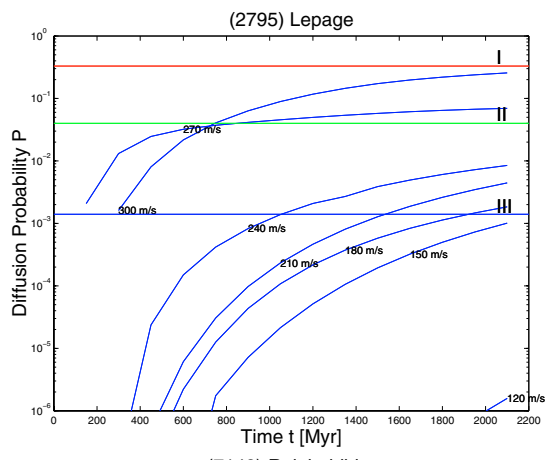

(7148) Reinholdbien

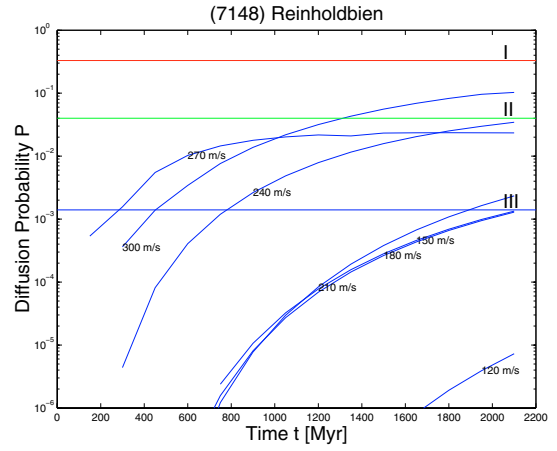

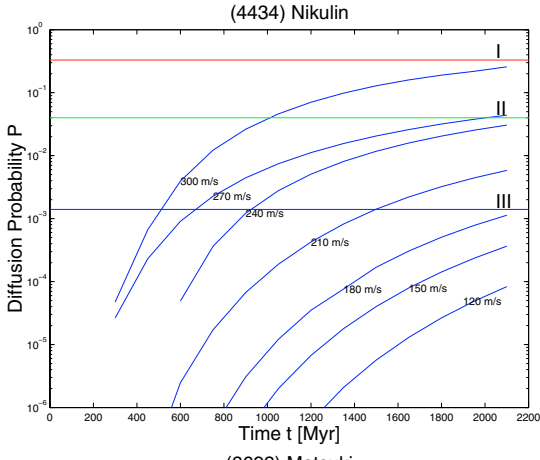

(8693) Matsuki

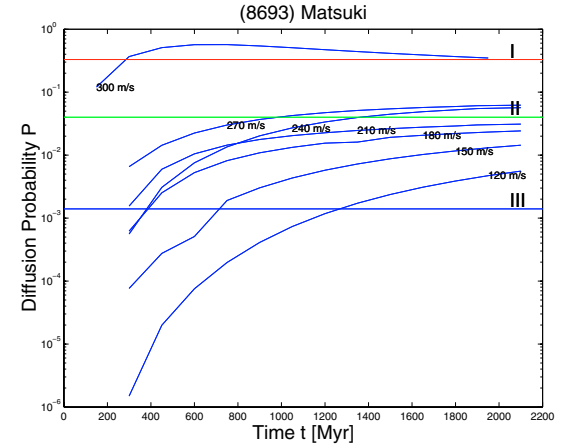

Fig. 14. The time evolution of the diffusion probabilities at the locations of the four V-type asteroids ((2795) Lepage, (4434) Nikulin, (7148) Reinholdbien, and (8693) Matsuki) most likely to be former family members scattered by (4) Vesta, for different values of $V$, the parameter defining our initial ejection velocity field. The horizontal lines display the diffusion probabilities associated with the four zones of diffusion at time $T$.

Table 5. The minimum time (in Myr) at which the probability levels of region III are reached for the first time for the four V-type asteroids we are considering, as a function of the initial ejection velocity $V_{\text {ej }}$.

\begin{tabular}{ccccccccc}
\hline \hline Ast. \# & Ast. name & $300 \mathrm{~m} / \mathrm{s}$ & $270 \mathrm{~m} / \mathrm{s}$ & $240 \mathrm{~m} / \mathrm{s}$ & $210 \mathrm{~m} / \mathrm{s}$ & $180 \mathrm{~m} / \mathrm{s}$ & $150 \mathrm{~m} / \mathrm{s}$ & $120 \mathrm{~m} / \mathrm{s}$ \\
\hline 2795 & Lepage & $>0$ & $>0$ & 1050 & 1500 & 1950 & & \\
4434 & Nikulin & 450 & 750 & 900 & 1500 & 2250 & & \\
7148 & Reinholdbien & 450 & 300 & 750 & 1800 & 2100 & & \\
8693 & Matsuki & $>0$ & $>0$ & 300 & $>0$ & 450 & 750 & 1350 \\
\hline
\end{tabular}

\section{Discussions}

In this work we studied the problem of the diffusion of members of an asteroid family via close encounters with the massive asteroid (4) Vesta. For this purpose we have:

1. Developed a new symplectic integrator, the São Paulo integrator, that is able to simulate the effect of both close encounters with a massive asteroid and the Yarkovsky effect.

2. Developed a new procedure based on the $p d f$ (probability density function, i.e., the function that describes the probability of having an encounter that modifies a proper element $x$ by $\Delta x$, for all the possible values of $\Delta x$ ), which is able to accurately estimate the probability that an ensemble of asteroids can diffuse at later times in a one-, two-, and threedimensional space. We believe our approach provides the first model that reproduces the long-term effect of repeated close encounters with some accuracy for the whole asteroidal distribution, instead that for a single moment, like in previous works (Nesvorný et al. 2002; Carruba et al. 2003).

3. Identified objects among the currently known V-type asteroids outside the Vesta family that we believe more likely to be explained as former members of the dynamical family that diffused mostly due to the effect of close encounters with (4) Vesta.

4. Estimated the minimum times necessary to scatter these objects to the current orbital positions, for different values of $V_{\text {ej }}$, a parameter identifying the initial possible values of the ejection velocity field.
Our results suggest that the Vesta family was formed with a relatively large initial ejection velocity field $\left(V_{\text {ej }}\right.$ of $((240 \pm 60) \mathrm{m} / \mathrm{s})$, and that the family is $(1200 \pm 700)$ Myr old.

As often happens in science, our work while answering many questions has left open several lines of research we believe worth pursuing. We consider the following projects a natural extension of this work:

1. In this paper we have determined the $p d f$ that best-fitted the results of diffusion for a fictitious family over two time intervals of $150 \mathrm{Myr}$ and over a limited region of space (roughly speaking, the one inside the $450 \mathrm{~m} / \mathrm{s}$ limit of maximum velocity at infinity). Two important questions remain unanswered: i) is the solution stable at later times (in other words, can we treat this process as a Markovian process of diffusion?) and ii) how does the $p d f$ vary as a function of the location of the asteroid in proper element space? Regarding the first question, preliminary results of an extension of our simulation to later times seem to suggest that the $p d f$ is indeed robust, and the problem of diffusion of an asteroid family can be treated as Markovian.

The second question is more demanding. It involves building our understanding of how the $p d f$ changes as a function of the asteroid locations. In Sect. 4 we started investigating this problem by trying to estimate how the change in heliocentric $\Delta U_{0}$ velocity depends on the impact parameter $b$ and relative velocity $V$. Logic would dictate that the $p d f$ shape (in particular the values of $e p_{\min }$ and $e p_{\max }$ of the exponential part) would depend on the maximum value of $\Delta U_{0}$, so that knowing in advance the expected values of relative 
velocities would in principle make it possible to determine the corresponding dependence on proper elements of the $p d f$. Of course, this is easier said than done, and prediction of such model would need to be extensively tested against the results of numerical simulations. This exceeds the purposes of this work, whose goal was to pioneer the field of modeling the long-term effects of close encounters with massive asteroids. But we believe it might be a very interesting subject for future research. Having a reliable diffusion model could allow us to make more accurate predictions of the Vesta family age, which is the other project we found very promising.

2. Based on the time evolution of diffusion probabilities at the locations of four asteroids that we believe were not likely to have been previously captured in powerful mean-motion or secular resonances, we set constraints on the Vesta family age and initial ejection velocity field. The approach we used is still in its initial stage, since we neglected both the Yarkovsky/YORP effect and possible passages through tiny mean-motion resonances. In a future work we are planning to refine our estimates further by including both effects in our model. We intend to follow (Vokrouhlický et al. 2006a,b) approach for modeling the Yarkovsky and YORP part, while extensive numerical simulation of the region of the four asteroids should provide information on the effects of passages of the bodies through the local web of resonances. Also, the existence of SLOAN V-type candidates may help us to further refine our estimate on the Vesta family age by allowing us to apply the procedure described in this paper to more objects. We encourage the observing community to obtain spectral identifications of these bodies, so as to eliminate any ambiguity inevitably associated with the photometric procedure used by Roig and Gil-Hutton (2006) in the attribution of spectral type.

When we started this project, it was generally believed that close encounters with massive asteroids were a minor effect, especially when compared to the Yarkovsky/YORP effect, and consequently were of little interest. Previous modeling efforts (including ours) were poor, and usually results were used to dismiss the role of this effect as a "noise" perturbing the semimajor axis evolution due to the Yarkovsky effect.

While the modeling of the long-term effects of close encounters is an interesting problem in itself, we have tried to show that the possible applications of such models, like the chronology of the Vesta family explored in this work, are promising and, in our opinion, worth exploring. We hope this line of research, while still in its infancy, may stir some interest in the role that massive asteroids may have played in the long-term evolution of the asteroid belt.

Acknowledgements. We wish to thank the anonymous reviewers for their careful revision of this article. We are grateful to Julio Klafke for helpful discussions of the modeling of scattering of asteroid via close encounters, and to Ricardo Cordeiro for sharing with us his work on anomalous diffusion in first-order jovian mean-motion resonances and for his insights into stochastic processes. This work was supported by the FAPESP (grants 03/07462-8, 06/50005-5) and CNPq funding agencies.

\section{References}

Alvarez-Candal, A., Lazzaro, D., \& Michtchenko, T. 2006, A\&A, submitted Asphaug, E. 1997, Meteor. Plan. Sci., 32, 965

Bachelier, L. 1900, Thesis, Sorbonne University, Paris, France

Bachelier, L. 1900, Théorie de la spéculation (Paris, France: Gauthier-Villars)
Bendjoya, P., \& Zappalá, V. 2002, in Asteroids III (Tucson, USA: Arizona University Press), 613

Biesiadecki, J. J., \& Skeel, R. D. 1993, J. Comput. Phys., 109, 318

Bottke, W. F., Nolan, M. C., Greenberg, R., \& Kolvoord 1993, Icarus, 107, 255

Bottke, W. F., \& Greenberg, R. 1994, Geophys. Res. Ltrs., 20, 879

Bottke, W. F., Vokrouhlickỳ, D., Brož, M., Nesvornỳ, D., \& Morbidelli, A. 2001, Science, 294, 1693

Brož, M. 1999, Thesis, Charles Univ., Prague, Czech Republic

Bus, J. S., \& Binzel, R. P. 2002, Icarus, 158, 146

Čapek, D., \& Vokrouhlickỳ, D. 2004, Icarus, 172, 526

Carpino, M., Milani, A., \& Nobili, A. 1987, A\&A, 181, 182

Carruba, V., Burns, J. A., Bottke, W., \& Nesvornỳ, D. 2003, Icarus, 162, 308

Carruba, V., Nesvornỳ, D., Burns, J. A. Ć. M., \& Tsiganis, K. 2004, AJ, 128, 1899

Carruba, V., Michtchenko, T., Roig, F., Ferraz-Mello, S., \& Nesvornỳ, D. 2005, A\&A, 441, 819

Cordeiro, R. R., \& Mendes de Souza, L. A. 2005, A\&A, 439, 375

Cruikshank, D. P., Tholen, D. J., Hartmann, W. K., \& Bell, J. F. 1991, Icarus, 89, Danby, J. M. A. 1988, Fundamentals of Celestial Mechanics, 2nd ed. (Willmann-Bell, Richmond, VA, USA)

Duffard, R., Lazzaro, D., Licandro, J., De Sanctis, M. C., Capria, M. T., \& Carvano, J. M. 2004, Icarus, 171, 120

Farinella, P., \& Davis, R. 1992, Icarus, 97, 111

Farinella, P., Vokrouhlickỳ, \& Hartmann, W., K. 1998, Icarus, 132, 378

Florczak, M., Lazzaro, D., \& Duffard, R. 2002, Icarus, 159, 178

Greenberg, R. 1982, AJ, 87, 184

Hughes, B. 1995, Random Walks and Random Environments, Vol. I (Oxford Science Publications)

Ivezič, Z., \& 30 colleagues 2001, Astron. J. 122, 2749

Knežević, Z., \& Milani, A. 2000, Celest. Mech. Dyn. Astron., 78, 17

Knežević, Z., \& Milani, A. 2003, A\&A, 403, 1165

Laskar, J. 2005, Proc. of CELMEC, 4, 16

Lazzaro, D., Michtchencko, T., Carvano, J. M., et al. 2000, Science, 288, 2033

Lazzaro, D., Roig, F., Michtchenko, T. A., \& Duffard, R. 2003, BASBr, 23, 241

Levison, H. F., \& Duncan, M. J. 1994, Icarus, 108, 18

Levison, H. F., \& Duncan, M. J. 2000, AJ, 120, 2117

Marzari, F., Cellino, A., Davis, D. R., Farinella, P., \& Vanzani, V. 1996, A\&A, 316,248

McCord, T. B., Adams, J. B., \& Johnson, T. V. 1970, Science, 168, 1445

Michtchenko, T., A., Lazzaro, D., Ferraz-Mello, S., \& Roig, F. 2002, Icarus, 158, 343

Milani, A., \& Nobili, A. M. 1984, Nature, 310, 753

Milani, A., \& Kneževič, Z. 1992, Icarus, 98, 211

Milani, A., \& Kneževic̀, Z. 1993, Icarus, 107, 219

Migliorini, F., Morbidelli, A., Zappalà, V., et al. 1997, Meteoritics \& Planetary Science, 32, 903

Morbidelli, A. 1993, Icarus, 105, 48

Morbidelli, A., \& Henrard, J. 1991, Cel. Mech. Dyn. Astr., 51, 169

Morbidelli, A., \& Vokrouhlický 2003, Icarus, 163, 120

Mothè-Diniz, T., Roig, F., \& Carvano, J. M. 2005, Icarus, 174, 54

Nesvornỳ, D., \& Morbidelli, A. 1998, AJ, 116, 3037

Nesvornỳ, D., \& Morbidelli, A. 1999, Celest. Mech. Dyn. Astron., 71, 243

Nesvornỳ, D., Morbidelli, A., Vokrouhlický, D., Bottke, W., \& Brož, M. 2002, Icarus, 157, 155

Nobili, A. M., Milani, A., \& Carpino, M. 1989, A\&A, 210, 313

Poincaré, H. 1897, Bull. Astron., 14, 53

Roig, F., \& Gil-Hutton, R. 2006, Icarus, submitted

Rubincam, D. P. 2000, Icarus, 2000, 114

Šidlichovskỳ, M., \& Nesvornỳ, D. 1997, Cel. Mech. Dyn. Astron., 65, 137

Takács, L. 1962, Processus stochastiques, problémes et solutions (Paris, France: Dunod)

Thomas, P. C., Binzel, R. P., Gaffey, M. J., et al. 1997, Icarus, 128, 88

Vokrouhlickỳ, D. 1999, AJ, 116, 2032

Vokrouhlickỳ, D., \& Čapek 2002, Icarus, 159, 449

Vokrouhlickỳ, D., Brož, M., Morbidelli, A., et al. 2002, Proc. ACM 2002

Vokrouhlickỳ, D., Brož, M., Morbidelli, A., et al. 2006a, Icarus, 182, 92

Vokrouhlickỳ, D., Brož, M., Bottke, W. F., Nesvorný, D., \& Morbidelli, A. 2006b, Icarus, 182, 118

Wisdom J., \& Holman, M. 1991, AJ, 102, 1528

Williams, J. G., \& Faulkner, J. 1981, Icarus, 46, 390

Wisniewski, W. Z. 1991, Icarus, 90, 117

Xu, S., Binzel, R. P., Burbine, T. H., \& Bus, S. J. 1995, Icarus, 115, 1

Zappalà V., Cellino, A., Farinella, P., \& Kneževič, Z. 1990, AJ, 100, 2030

Zappalà, V., Bendjoya, Ph., Cellino, A., Farinella, P., \& Froeschlè, C. 1995, Icarus, 116, 291 
V. Carruba et al.: Modeling close encounters with massive asteroids, Online Material $p 1$

\section{Online Material}




\section{Appendix A: definitions of mean-motions and secular resonances}

Mean-motion resonances are resonances that involve the meanmotion of an asteroid and one (two-body resonance) or more (three-body, four-body, etc. resonance) planet, such that the mean-motions satisfy the relationship

$m_{\mathrm{J}} \dot{\lambda}_{\mathrm{J}}+m_{\mathrm{S}} \dot{\lambda}_{\mathrm{S}}+\ldots+m_{\mathrm{A}} \dot{\lambda}_{\mathrm{A}} \simeq 0$

where $\lambda$ denotes the mean-motions and the suffixes J, S, A stand for Jupiter, Saturn (or other planets), and asteroids, respectively; $m_{\mathrm{J}}, m_{\mathrm{S}}, \ldots, m_{\mathrm{A}}$ are integers. The resonant arguments associated with such resonances turn out to be any combinations of

$\sigma=\sum_{i}\left(m_{i} \lambda_{i}+p_{i} \varpi_{i}+q_{i} \Omega_{i}\right)=0$

where $i$ is a suffix that indicates either the planets ( $\mathrm{J}$ for Jupiter, $\mathrm{S}$, for Saturn, etc.) or the asteroid, and $\varpi$ and $\Omega$ are the longitudes of pericenter and nodes, respectively. The coefficients $m_{i}, p_{i}, q_{i}$ have to fulfill the D'Alembert rules of permissible arguments; i.e., the sum of coefficients of individual longitudes must be zero, and the sum of coefficients of nodal longitudes must be even.

By secular resonances we mean a resonance between the frequency of variation in the longitudes of perihelia $\left(g_{i}=\dot{\varpi}_{i}\right)$, in the longitudes of nodes $\left(s_{i}=\dot{\Omega}_{i}\right)$, or both. The coefficients for such resonances must still satisfy D'Alembert rules.

\section{Appendix B: determining the optimal pdf}

For brevity, we only describe the procedure for obtaining the $p d f$ for proper $e$. The procedure is analogous for the $p d f \mathrm{~s}$ in proper $a$ and $i$. To best determine the parameters of the $p d f$ we applied Eq. (5) to the initial distribution in $e$ of our asteroids with different parameters for $t=T, 2 T$, where $T$ varied from $100 \mathrm{Myr}$ to 200 Myr. To quantitatively estimate how the propagated distributions approximate the real ones, we defined an $\chi^{2}$-like variable of the form:

$\chi^{2}=\sum_{i}\left(\frac{p_{i}-d_{i}}{d_{i}}\right)^{2}$,

where $p_{i}$ is the value of the propagated distribution for the ith interval, while $d_{i}$ is the value of the real distribution for the same interval ${ }^{11}$. To confront the propagated and real distributions using Eq. (13) we fitted a Gaussian to the real data. Since the original distribution and the simulated ones already followed Gaussian distributions, we believe this procedure is justifiable (otherwise, we would have to deal with the problem of bestfitting the continuous distribution produced by Eq. (5) with the discontinuous distribution of number of real asteroids per bin in proper element).

Figure B.1 shows the typical results of the application of our model, together with the distribution of $e$ produced by numerical simulations. When applying Eq. (13) values of $\chi^{2}$ that are much lower than the number of degrees of freedom are a proof of a good fit and can be associated to a maximum probability that the two distributions are different (Carruba et al. 2004). However, rather than knowing a single value of $\chi^{2}$, the knowledge of where the minimum of $\chi^{2}$ is located for a distribution of values of free

\footnotetext{
${ }^{11}$ To avoid the problem of small divisors in Eq. (13), we eliminated all the cases with $d_{i}<10^{-5}$. This typically left us with $\simeq 100$ intervals, or degrees of freedom.
}
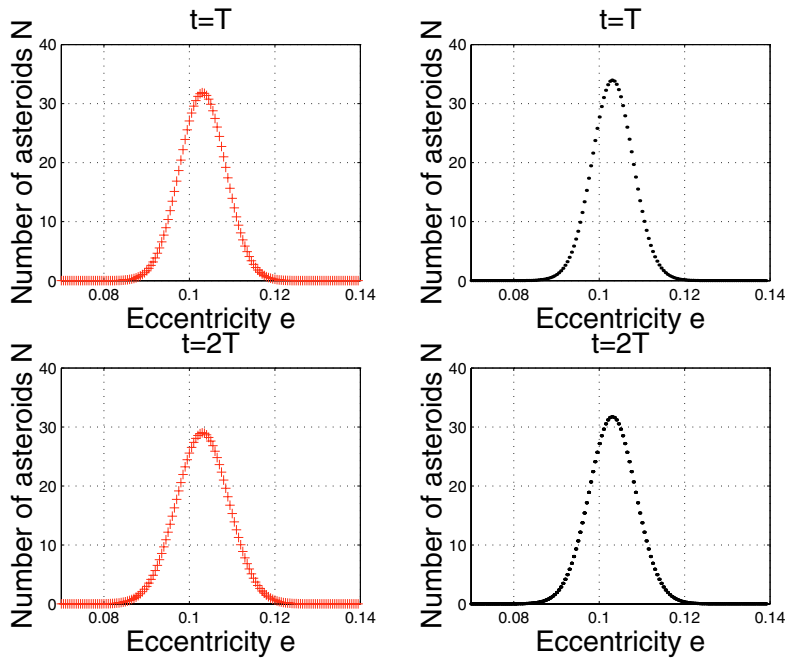

Fig. B.1. Real (red + signs, left panels) and propagated (black dots, right panels) distributions for times $t=T$ (top panels) and $t=2 T$ (bottom panels). The $\chi^{2}$ values of the differences between the two distributions was 0.9149 and 0.5552 (Eq. (13)) ( $\simeq 100$ degrees of freedoms), respectively, which indicates a very good fit.

parameters may provide information on the set of parameters that best fits the observed distribution. This method has been recently applied by Vokrouhlický et al. (2005) to determine the age and the components of the ejection velocity fields from the evolution in proper $a$ due to the Yarkovsky and YORP effect of the Eos and other asteroid families.

We start by analyzing the dependence of the fit on $\sigma$, the standard deviation of the Gaussian part of the $p d f$. We used $e_{\min }=0.0045, e_{\max }=0.0105, s=12$, and $T=150 \mathrm{Myr}$, and let $\sigma$ vary from 0.0009 to 0033 (the reason behind the choice of this parameters will be justified later in this section). The left panel of Fig. B.3 shows how the values of $\chi^{2}$ vary for the fitted distributions at $t=T$ and $t=2 T$. This second output is obtained by first propagating the real distribution with Eq. (5) and by then propagating this output over another interval $T$, and the second output is then compared with the data produced by the numerical simulation. We chose to best-fit data over two time intervals $T$ in order to obtain a $p d f$ that is as independent as possible from stochastic fluctuations on the number of encounters over a time $T$. We believe that in this way the $p d f$ we found can be more reliably used when the extrapolation of our result is considered (see Sect. 5).

All values of $\chi^{2}$ are excellent for $\sigma$ in the range from 0.0013 to 0.0023 , which shows how the $p d f$ is actually only weakly dependent on the aperture of the normal part of the distribution. The minima of the two distributions and the value of the parameter we chose for our model occur at $\sigma=0.0012$, which corresponds to the standard deviation of the real distribution of variations in proper $e$ due to close encounters, when the largest changes due to encounters occurring at very low velocities and minimal distances from (4) Vesta were not considered, and is therefore a very realistic value to use.

The next step is to determine the parameters of the exponential part of the $p d f$. We started by trying to constrain the values of $e_{\min }$ and $e_{\max }$. For this purpose we applied Eq. (5) for a grid of values in $e_{\min }$ and $e_{\max }$ from 0.0035 to 0.0075 and from 0.0090 to 0.0130 , respectively, in steps of 0.0005 . This range of values of $e_{\min }$ and $e_{\max }$ is compatible with the observed distributions of the encounters that caused the largest variation in proper $e$. 

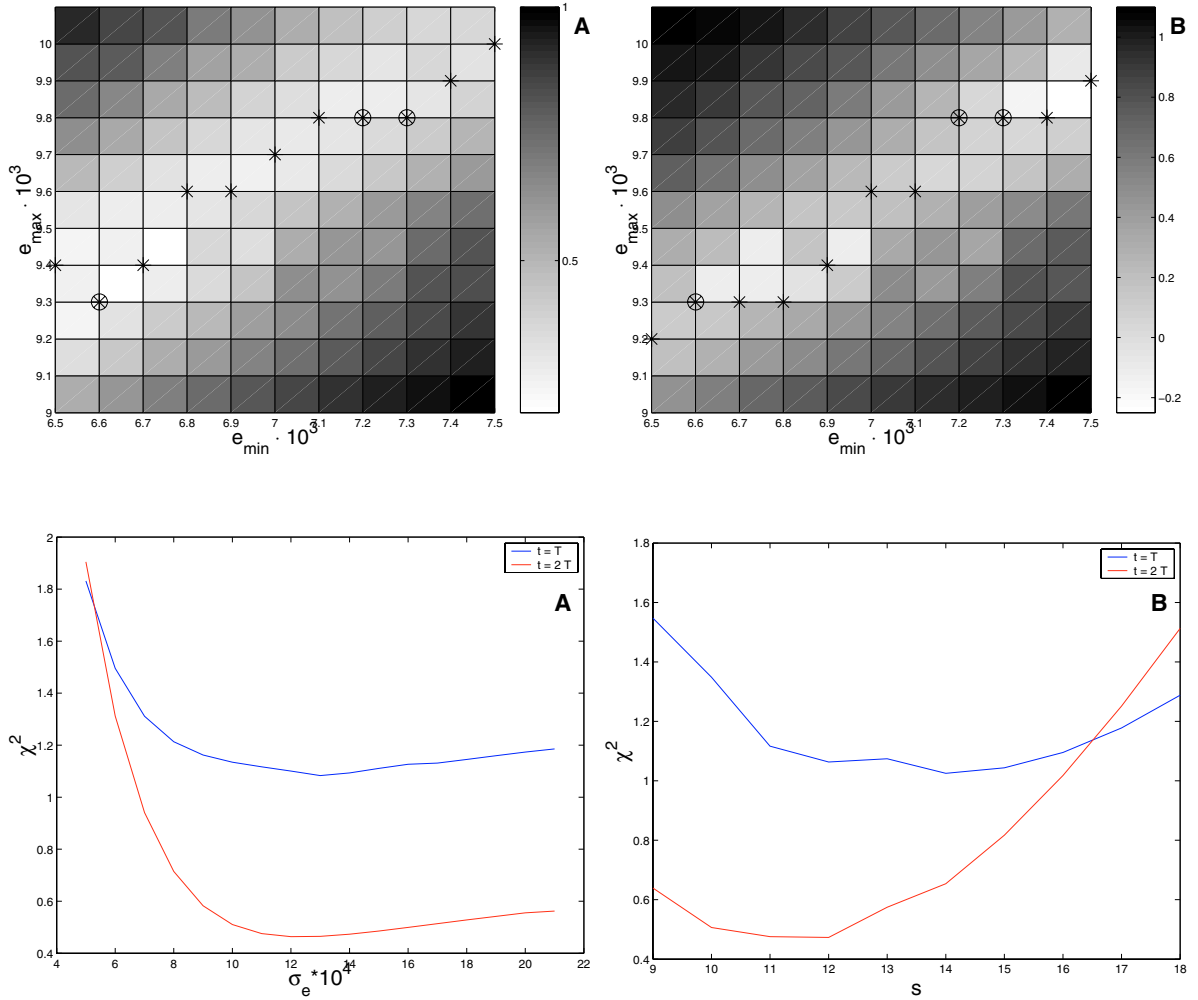

Fig. B.2. Color plot of $\log _{10}\left(\chi^{2}\right)$ for $t=$ $T$ (left panel) and $t=2 T$ (right panel), as a function of $10^{3} \times e_{\min }$ vs. $10^{3} \times e_{\max }$. The lowest values of $\chi^{2}$ (best fit) are in whiter tones in the figures. The asterisks show the locations of the local minimum of $\chi^{2}$, while the circles identify the minima common to the $t=T$ and $t=2 T$ solutions.

Fig. B.3. Dependence of $\chi^{2}$ as a function of $\sigma$ (left panel) and $s$ (right panel). For $\sigma$ and $s$ we took the values corresponding at the common minima of the best fit curves for $t=T$ and $t=2 T$.

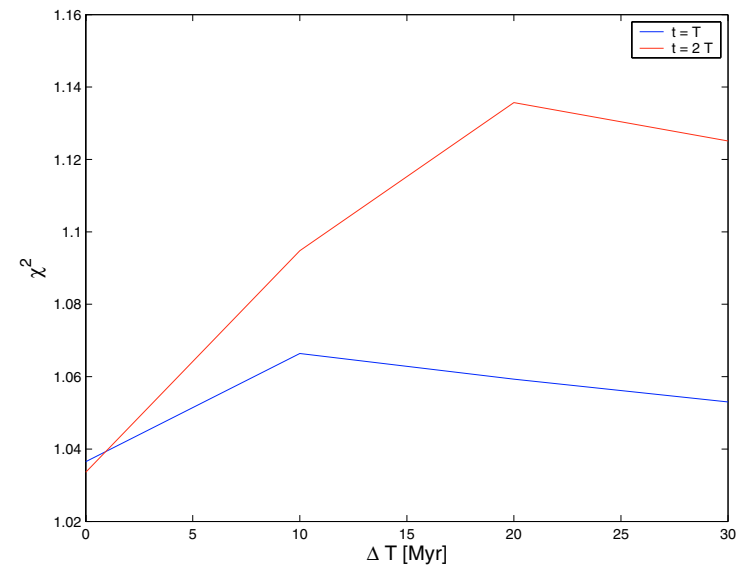

Fig. B.4. Dependence of $\chi^{2}$ as a function of $\Delta T$, with $\Delta T$ going from 0 to $30 \mathrm{Myr}$, for the $p d f$ found in Sect. 3.2.

Figure B.2 shows the color plots of $\log _{10}\left(\chi^{2}\right)$ values for $t=T$ and $t=2 T$. The first thing we notice is that there is not a unique solution, but several that tend to be aligned along the line $e_{\max }-e_{\min } \simeq 0.0055$. A solution that best fits the distribution at $t=T$ is not necessarily a best fit for $t=2 T$ (but they are usually very close). Solutions for $e_{\min }>0.005$ produce best fits in $\chi^{2}$, but are not compatible with the results of the numerical simulations, which show a maximum value of $e$ for the normal part of 0.0045 . We identified three common solutions in the allowed range and chose to work with the one that had the lowest value of $\chi^{2},\left(e_{\min }=0.0045\right.$ and $\left.e_{\max }=0.0105\right)$, and whose values of $e_{\min }$ and $e_{\max }$ were more compatible with the observed distribution.

The next parameter that needed to be set was $s$. We applied a procedure similar to the one used for $\sigma$ (see Fig. B.3, right panel) and found that i) as for the case of $\sigma$, the fit is not strongly de- pendent on the value of $s$-essentially all values of $s$ in the range between 11 and 17 produced results with excellent $\chi^{2}$ values and ii) while there was a single minimum for the distribution of $\chi^{2}$ at $t=2 T$, there were two local minima at $t=T$. We chose the value for which the local minimum at $t=T$ was equal to the minimum at $t=2 T$, i.e., $s=12$.

Finally, we needed to understand for what value of $T$ the set of registered close encounters was large enough to allow the determination of a $p d f$ that would allow a prediction of asteroid proper elements that would not change significantly for higher $T$. In fact, at some point in time, all possible encounter geometries should have already happened, and the $p d f$ computed for interval $T$ should not produce significantly different results from the one computed for $T+\Delta T$. The best way to verify this should be by computing several $p d f$ functions for different time intervals $T$ and choosing the optimal value of $T$ as the one for which the fits produced by successive $p d f$ s do not differ significantly.

This procedure, while quite accurate, is however rather cumbersome. It involves doing several parametric studies like the one above described for different $T \mathrm{~s}$, which requires a considerable investment in time. In this work, we prefer to use a different approach. Given a time T long enough to have a significant sample of encounters at low relative distances and velocities, which previous experiences (Carruba et al. 2003) indicate are in the order of $150 \mathrm{Myr}$, we compute a $p d f$ with the procedure described above. We then try to fit the results using the same $p d f$, but for different times $T$. If the set of close encounters is complete enough, then the shape of the $p d f$ should remain essentially the same, and the same $p d f$ should be able to reproduce the results for $t$ higher than $T$, with an error depending on the number of encounters that happened between $T$ and $T+\Delta T$. In particular, trends in the values of $\chi^{2}$ can give us information about the goodness of the fit and the validity of our approximation.

Figure B. 4 shows the values of $\chi^{2}$ for $T=150 \mathrm{Myr}$ and $\Delta T$ going from 0 to $30 \mathrm{Myr}$. First, since $\chi^{2}$ does not vary by more than $11 \%$, we consider our choice of $p d f$ as rather robust. We do 
V. Carruba et al.: Modeling close encounters with massive asteroids, Online Material $p 4$

not observe a single trend in the value of $\chi^{2}$, but instead a variation probably caused by the stochastic nature of the encounters. A study for higher values of $\Delta T$ could in principle evidence if such trend does exist, but unfortunately the time range of our simulations does not allow us to go beyond $\Delta T=30 \mathrm{Myr}$. We believe that studying the dependence of $\chi^{2}$ on $\Delta T$ should certainly be the subject of future research.

Now that all parameters for the $p d f$ in $e$ are determined, we applied the same procedure described in this section to the distribution in proper $a$ and $i$. Results for the three $p d f$ s are summarized in Table 3. 